Journal for ImmunoTherapy of Cancer

\section{NK cells enhance CAR-T cell antitumor efficacy by enhancing immune/tumor cells cluster formation and improving CAR-T cell fitness}

To cite: Bachiller M, PerezAmill L, Battram AM, et al. NK cells enhance CAR-T cell antitumor efficacy by enhancing immune/tumor cells cluster formation and improving CAR-T cell fitness. Journal for ImmunoTherapy of Cancer 2021;9:e002866. doi:10.1136/ jitc-2021-002866

- Additional supplemental material is published online only. To view, please visit the journal online (http://dx.doi.org/10. 1136/jitc-2021-002866).

MB and LP-A contributed equally.

Accepted 28 July 2021

\section{ABSTRACT}

Background Chimeric antigen receptor (CAR)-T cell immunotherapy has modified the concept of treatment in hematological malignancies. In comparison with pediatric patients, where responses are maintained over many years, older patients, such as those with non-Hodgkin's lymphoma (NHL) and multiple myeloma (MM), present lower persistence of CAR-T cells that might be due to decreased fitness of $\mathrm{T}$ cells acquired with aging. Moreover, cord blood derived-NK cells (CB-NKs) and CAR-NK cells derived from CB-NK can be used 'off-the-shelf' as immune cells with antitumor properties for the treatment of cancer patients. However, to date, clinical studies have only demonstrated the safety of these therapies but not optimal efficacy. To confront the shortcomings of each therapy, we devised a novel approach consisting of simultaneous (CAR-)NK cell and CAR-T cell administration. In this setting, NK cells demonstrate an important immunoregulation of T cells that could be exploited to enhance the efficacy of CAR-T cells.

Methods A combinatorial treatment based on either CAR-T and CAR-NK cells or CB-NK and CAR-T cells in two models of NHL and MM was performed. Antitumor efficacy was analyzed in vitro and in vivo, and parameters related to early activation, exhaustion and senescence of $T$ cells were analyzed.

Results We show that CAR-NK cells derived from CB-NK are only effective at high doses (high E:T ratio) and that their activity rapidly decreases over time in comparison with CAR-T cells. In comparison and to exploit the potential of 'off-the-shelf' CB-NK, we demonstrate that a low number of CB-NK in the CAR-T cell product promotes an early activation of CAR-T cells and their migration to MM cells leading to enhanced anti-MM efficacy. Moreover, cytokines related to CRS development were not increased, and importantly, CB-NK enhanced the fitness of both $\mathrm{CAR}^{\text {pos }}$ and $\mathrm{CAR}^{\text {neg }} \mathrm{T}$ cells, promoting lower levels of exhaustion and senescence.

Conclusion This study demonstrates a relevant immunoregulatory role of CB-NK collaborating with CAR-T cells to enhance their antitumor activity. A novel and different approach to consider in CAR-T cell immunotherapy studies is presented here with the goal to enhance the efficacy of the treatment.

\section{INTRODUCTION}

In recent years, adoptive cell immunotherapy approaches, particularly administering chimeric antigen receptor (CAR)-modified $\mathrm{T}$ cells, have been established as new methods of treatment for patients with hematological malignancies. ${ }^{\text {l }}$ To date, most clinical studies in patients with $\mathrm{B}$ cell malignancies administer CAR-T cells in an autologous setting. ${ }^{23}$ However, there is a proportion of patients where the production of CAR-T cells results in an insufficient amount required for the treatment, and unfortunately, patients do not receive the therapy. For this type of patient, a universal source of immune cells, such as CAR-modified natural killer (NK) cells, are becoming an attractive option. However, whereas CAR-NK cells have demonstrated superiority over unmodified NK cells in preclinical studies ${ }^{45}$ and safety in clinical studies, ${ }^{6}$ their efficacy is still lower than that of CAR-T cells. ${ }^{17}$

Specifically, CAR-T cells directed to CD19 (CART-19) have achieved outstanding responses in acute lymphoblastic leukemia (ALL) and non-Hodgkin's lymphoma (NHL) patients, ${ }^{3-10}$ and B-cell maturation antigen (BCMA) has emerged as the most promising target for CAR-T cells used to treat multiple myeloma (MM) patients. $^{11-16}$ However, whereas pediatric ALL patients treated with CART-19 cells maintain durable responses that correlate with CAR-T cell persistence, ${ }^{3}$ NHL patients present poorer responses. ${ }^{10}$ Moreover, in MM patients, CART-BCMA cells do not persist long, and patients end up relapsing despite achieving initial complete responses. $^{15} 16$ These findings indicate the need to improve CAR-T cell persistence and efficacy in NHL and MM patients to avoid relapses. 
Cord blood-derived NK cells (CB-NKs) have been established as a source to obtain 'off-the-shelf' NK cells for the treatment of cancer patients. ${ }^{17} 18$ CAR-NK cells obtained from CB-NK have demonstrated improved antitumor activity compared with unmodified CB-NK ${ }^{5}$ and have also demonstrated safety in patients. ${ }^{619}$ We previously observed that when CB-NK come into contact with MM tumor cells, they secrete a variety of proinflammatory molecules that enhance the formation of tumor/immune cell clusters, bringing $\mathrm{T}$ lymphocytes and other immune cells into close contact and enhancing the antitumor activity of $\mathrm{T}$ lymphocytes. ${ }^{20}$ Therefore, we hypothesized that NK cells, either CB-NK or CAR-NK cells, would enhance the efficacy of CAR-T cells leading to more durable responses.

Here, we first confirmed that at low immune cell numbers, CAR-T cells are superior to CAR-NK cells. Then, to enhance the efficacy of CAR-T cells, we designed a combinatorial treatment based on CAR-T cells with either CAR-NK cells or CB-NK. Two CARs directed against CD19 $(\mathrm{ARI}-0001)^{2}$ and BCMA (ARI2h), ${ }^{14}$ which were previously developed by us and which have been administered to patients, were employed. We demonstrate that a combinatorial treatment for NHL with CART-19 and CARNK-19 cells does not improve the efficacy compared with the same total number of CART-19 cells alone, suggesting that there is a minimum number of CAR-T cells that is critical for the antitumor efficacy. However, a combinatorial treatment based on CAR-T cells with the addition of half the dose of CB-NK enhances the antitumor efficacy in a model of MM and ARI2h cells. This beneficial impact of CB-NK over ARI2h cells is initiated at incredibly early time points of tumor/immune cell contact where CB-NK facilitate earlier activation and enhanced migration of ARI2h cells to tumor cells. These events impact on the fitness of ARI2h cells in the long term by ameliorating exhaustion levels and the emergence of markers related to immunosenescence in ARI2h cells.

\section{METHODS}

\section{Donors of immune cells}

CB-NK and peripheral blood $\mathrm{T}$ cells were obtained from the Banc de Sang i Teixits de Barcelona (BST) from either cord blood (CB) or peripheral blood from healthy donors after obtaining informed consent and being approved by the BST.

\section{Tumor cell lines}

Ramos, RPMI8226, U266 and K562 cell lines were purchased from American Tissue Culture Collection (Manassas, Virginia, USA). ARP1 cell line was kindly provided by Multiple Myeloma Research Center (Little Rock, Arkansas, USA). RPMI8226, K562 and ARP1 were cultured in RPMI with $10 \%$ fetal bovine serum (FBS) and $1 \%$ penicillin/streptomycin (Pen/Strep) and U266 with $15 \%$ FBS. K562-based artificial antigen-presenting cells expressing membrane-bound Interleukin (IL)-21 (K562-mb21-41BBL feeder cells) were cultured in RPMI with $10 \%$ FBS and 1\% Pen/Strep. Tumor MM cell lines were modified to express GFP-FireFlyLuciferase (GFPFFLuc) using the plasmids pLV-MSCV_Luc-T2A-GFP (kindly provided by Amer Najjar) coding for GFP-FFLuc, and pMD2.G and psPAX2 coding for VSV-G and gag/ pol, respectively. Mycoplasma testing in cell lines was performed every 2 months.

\section{Lentivirus production for CAR-T and CAR-NK cells}

For CAR-T cells, either pCCL-EF1a-CAR19 or pCCLEFla-CARBCMA with packaging plasmids pMDLg-pRRE, pRSV-Rev and envelope plasmid VSV were used. For CAR-NK cells, pCCL-MSCV-CAR19 with pMDLg-pRRE, pRSV-Rev and envelope plasmid BaEV-TR (provided by Dr Els Verhoeyen) were used for production of virus particles. Production of virus particles to transduce T cells was done in HEK293T cells using the same protocol previously described by us. ${ }^{14}$ Production of virus for CAR-NK cells was done in HEK293T cells that were transfected with transfer vector pCCL-MCSV-CAR19 with pMDLg-pRRE, pRSV-Rev and BaEV-TR plasmid. JetPEI (PolyPlus Transfection) was used as a transfection reagent. Transfection was performed using $\mathrm{NaCl}$ and JetPei following the manufacturer's protocol. Viral supernatants were collected at 48 hours and concentrated with Lenti-X Concentrator (ClonTech) following the supplier's protocol and stored at $-80^{\circ} \mathrm{C}$ until use.

\section{CB-NK expansion}

K562-mb21-41BBL feeder cells were used to expand untransduced NK cells. They were irradiated at $100 \mathrm{~Gy}$ and added at a feeder cell:CB-NK ratio of 2:1. CB-NK were left to expand for 14 days, with new feeder cells added on day 7 .

\section{T cells and CB-NK transduction}

$\mathrm{T}$ cells were obtained from buffy coats by Ficoll and magnetic $\mathrm{T}$ cell depletion (Miltenyi Biotec). T cells were expanded in Click's media (50\% RPMI, 50\% Click's (Irvine Scientific), 5\% human serum, 1\% Pen/Strep), activated with Dynabeads Human T-Activator CD3/CD28 (Thermo Fisher Scientific) and IL-2 (100 IU/mL) every other day. Experiments were performed after 8-10 days of $\mathrm{T}$ cell expansion. Viral transduction was performed after 48 hours of the expansion at a MOI of 10 . NK cells were selected from $\mathrm{CB}$ units by Ficoll and magnetic NK cell depletion (Miltenyi Biotec). To produce CAR-NK (ARI3 cells), NK cells from a CB unit were expanded with NK MACS Medium (Miltenyi Biotec), $1 \%$ NK MACS Supplement, 5\% human serum, IL-2 $(500 \mathrm{IU} / \mathrm{mL})$ and IL-15 $(140 \mathrm{IU} / \mathrm{mL})$. On day 5 , CB-NK were transduced at a multiplicity of infection (MOI) of 5 with Vectofusin-1 $(10 \mathrm{mg} / \mathrm{mL})$ following Vectofusin-1 protocol (Miltenyi Biotec). Cells were washed 6-24hours after transduction. On day 7, ARI3 cells were coexpanded with feeder cells adding IL2 $(400 \mathrm{IU} / \mathrm{mL})$ every other day for the next 7 days. Of note, throughout the manuscript, 'ARI3 cells' 
refers to CAR-NK cells and CB-NK refers to untransduced NK cells.

\section{In vivo models}

Immunodeficient NSG mice were purchased from The Jackson Laboratory. Mice used for experiments were of the same sex and 8-12 weeks old. Mice were irradiated on day 1 at a dose of $2 \mathrm{~Gy}$ in a biological radiator with two sources of 137 Cs (J.L. Shepherd, model MARK 1, 30 serial number 1199) and received itravenous tumor cells modified to express GFP-FFLuc on day 0. Different models of tumor burden were created for NHL depending on the dose of NHL cells administered. Thus, mice received $1 \times 10^{5}, 3.5 \times 10^{5}$, or $5 \times 10^{5}$ NHL cells to create the low, medium, or high tumor burden models. Then, they received immune cells on day 7 (for NHL) or day 14 (for MM). The disease was followed weekly by bioluminescence as previously described.$^{20}$ Bone marrow (BM) and spleen were harvested when mice were euthanized to analyze the presence of CAR-T cells and MM cells by flow cytometry.

\section{Cytotoxicity assays}

Luciferase killing assays were performed by coculturing target tumor cells expressing GFP-FFLuc with effector cells tested in each case in a white-walled 96-well plate. D-luciferin $(20 \mathrm{mg} / \mathrm{mL})$ was added $15 \mathrm{~min}$ prior to the bioluminescence reading in a Synergy HT Plate Reader (BioTek). The percentage of remaining tumor cells was calculated as (luminescence of sample/luminescence of tumor cells alone) $\times 100$.

\section{Cytokine production}

Interferon Gamma (IFN $\gamma$ ), tumor necrosis factor alpha $(\mathrm{TNF} \alpha)$ and IL-2 were quantified by ELISA (ELISA MAX Deluxe Set, Biolegend) following the manufacturer's protocol. Luminex assays were conducted with ProcartaPlex multiplex immunoassay kit (Thermo Fisher Scientific).

\section{Confocal microscopy}

RPMI8226 cell line modified to express GFP was cocultured with CART cells stained with Cell Tracker Blue CMAC Dye (Thermo Fisher Scientific). Either CB-NK or non-transduced T cells (UT) were stained with CellTracker Deep Red Dye (Thermo Fisher Scientific) and added to the previously mentioned coculture. Images were acquired using a Leica SP5 microscope. 405, 488 and 633 lasers were used for excitation. For time lapse experiments, in vivo image acquisitions were performed every $30 \mathrm{~s}$ for 14 hours.

\section{Challenges}

ARP1-GFP-FFLuc (MM) cells were cocultured with CART cells at an effector:target ratio 0.5:1. In the condition with CB-NK, CB-NK were added at half the amount of CART cells, leaving a final ratio of CART:MM(:NK) 0.5:1(:0.25). The remaining tumor cells were assessed by fluorescent microscopy until no GFP was observed, and thus the challenge was over. T lymphocytes were then stained, and the phenotype was compared with that seen in unstimulated $\mathrm{T}$ cells (day 0 ) and CAR-T cells before exposure to tumor cells.

\section{Flow cytometry}

CAR expression was detected with either a recombinant BCMA-Fc protein (Enzo Life Sciences) or a recombinant CD19-Fc protein (Thermo Fisher Scientific) and an antihuman IgG Fc-BV-421 (Biolegend, clone: M1310G05). For T cell phenotyping, PD-1-APC (clone: J105), TIM3-FITC (clone: F38-2E2), TIGIT-PerCP-Cy5.5 (clone: A15153G), LAG-3-PE (clone: 11C3C65), CXCR3-AlexaFluor 488 (Clone 1C6/CXCR3), CCR7-PerCP-Cy5.5 (clone: 150503), CD45RA-APC (clone: HI100), CD27-PE (clone: M-T271), CD28-FITC (Clone CD28.2), CD3-PE (clone SK7), CD4-APC-H7 (clone L200) and CD8PE-Cy7 (clone: RPA-T8) antibodies were used. For NK cell phenotyping, CD16-Alexa488 (Clone 3G8), NKP30PeCy7 (Clone P30-15), NKG2D-APC-Cy7 (Clone 1D11), NKG2A-PacificBlue (Clone S19004C), NKG2C-Pe (Clone S19005E), KIR2DL1-PE (Clone HP-DM1), KIR2DL2/ L3-FITC (Clone DX27), KIR3DL1-BV421 (Clone DX9) and KIR2DL1/S1/S3/S5-PerCp Cy5.5 (Clone HP-MA4) were all from Biolegend. CD56-APC (Clone REA196, Miltenyi) was used for NK cells. Staining was performed in FACS buffer. For T cell determination in BM or spleen at in vivo end-point, mouse FcR blocking reagent (Miltenyi) was used. All experiments were read on a FACS Canto II (BD Biosciences) and analyzed with FlowJo software (Tree Star, Eugene, Oregon, USA).

\section{Graphs and statistical analysis}

Data were presented using GraphPad Prism software, and statistical analysis was performed using SPSS software V.20 using Student's t-test to compare between two groups.

\section{RESULTS}

CAR-NK cells demonstrate higher in vitro activity than CAR-T cells only at high E:T ratios, requiring IL2 to maintain efficacy at low E:T ratios, which can be provided by CAR-T cells

We first optimized a method to obtain a high number of CAR-NK cells directed against CD19 (ARI3 cells) starting from CB-NK. We used a CAR directed against CD19 with $4-1 \mathrm{BB}$ as costimulatory domain previously produced by our institution termed ARI-0001 (ARI1 from now on). ${ }^{21}$ Plasmids coding for ARI1 and ARI3 differed in the promoter (online supplemental figure 1A) as CB-NK presented higher transduction efficiencies using the MCSV promoter instead of the EF1a promoter. Another difference between ARI1 and ARI3 was the packaging plasmid that was used in the transduction. The use of a Vesicular Stomatitis Virus (VSV) packaging plasmid for virus production led to extremely low transduction efficiencies of CB-NK. Interestingly, Bari et $a l^{22}$ demonstrated that exchanging VSV for Baboon Envelope (BaEV) packaging plasmid 
improved efficiencies of NK cell transduction. Therefore, VSV and BaEV were compared confirming improved efficiency of CAR transduction with BaEV (online supplemental figure 1online supplemental figure 1B). Then, two different expansion methods were compared with $\mathrm{BaEV}$ (figure 1A). In the first method (A.1), CB-NK were expanded with IL2 and IL15 over 5 days. On day 5 , CAR transduction was performed. On day 7, K562-mb21-41BBL feeder cells were added to CB-NK, and they were left to expand until day 14 with the addition of IL2 every other day. In the second method (A.2), CB-NK were expanded with IL2 and feeder cells for 5 days. On day 5, CAR transduction was performed. On day 7 , CB-NK were left to expand until day 14 with the addition of fresh feeder cells and IL2 every other day. Method A.1 achieved a higher percentage of ARI3 + cells than method A.2 (figure 1B), and therefore, method A.1 was selected for all ARI3 cell productions.

The anti-NHL activity of both CAR-T cells and CAR-NK cells directed against CD19 (ARI1 for CAR-T cells and ARI3 for CAR-NK cells: online supplemental figure 1A was compared in vitro. At higher effector:target (E:T) ratios, ARI3 cells presented higher antitumor activity than untransduced CB-NK or ARI1 cells (figure 1C). However, at low E:T ratios, ARI3 efficacy was significantly lower to that of ARI1 cells (figure 1D), being this difference even more pronounced at longer times (24hours vs 48 hours). Lack of efficacy of NK cells at longer times might reflect their requirement for IL2, which is not present in these assays. Therefore, we confirmed that addition of exogenous IL2 improved the in vitro efficacy of ARI3 cells, especially at later time points (figure 1D). In terms of cytokine production, at higher E:T ratios, ARI3 cells produced a higher amount of IFN $\gamma$ than CB-NK, with this production being increased further with the addition of IL2. However, production of IFN $\gamma$ by ARI3 cells with and without IL2 was always lower than that of ARI1 cells (figure 1E). Regarding TNF $\alpha$, this cytokine is rapidly produced at early time points and decays fast. ${ }^{14}$ While ARI3 cells produced higher $\mathrm{TNF} \alpha$ levels than CB-NK, the highest TNF $\alpha$ production was detected for ARI1 cells (figure 1F).

ARI3 cells required IL2 at low E:T ratios to kill tumor cells, and interestingly, CAR-T cells produce high amounts of IL2. Moreover, we previously demonstrated that CB-NK can enhance the antitumor activity of untransduced (UT) $\mathrm{T}$ cells. ${ }^{20}$ Therefore, we hypothesized that ARI1 CAR-T cells would boost the activity of ARI3 CAR-NK cells, and vice versa, which would lead to an overall enhanced antitumor efficacy. Thus, combination of ARI1 and ARI3 cells, where the total number of immune cells was the same as ARI1 cells alone $(0.5+0.5$ vs 1$)$, demonstrated increased anti-NHL activity (figure 1G) without impacting in higher IFN $\gamma$ (figure $1 \mathrm{H}$ ) and TNF $\alpha$ production (online supplemental figure 1C). Of note, when the total number of immune cells combining ARI1 and ARI3 was double that of ARI1 cells alone (1+1 vs 1$)$, a higher anti-NHL activity and IFN $\gamma$ production (figure $1 \mathrm{G}$ and $\mathrm{H}$ ) was observed with hardly any changes for TNF $\alpha$ (online supplemental figure 1C). Last, long-term in vitro cytotoxicity assays performed with a low E:T ratio demonstrated superiority of this combinatorial treatment (ARI1 +ARI3) versus ARI1 cells alone when the total number of immune cells was the same in both treatments (figure 1I). Of note, IFN $\gamma$ levels were not increased (figure 1J).

\section{CAR-NK cells do not maintain in vivo efficacy and enhance the migration of tumor cells to secondary lymphoid tissues in a model of NHL}

The impact of IL2 in the activity of ARI3 cells and the combinatorial treatment were further evaluated in vivo in different NHL tumor burden models. In a model of highly aggressive NHL, none of the immune cells administered (CB-NK, ARI1 or ARI3) were able to prevent disease progression at all (online supplemental figure $2 \mathrm{~A}$ and $2 \mathrm{~B}$ ). Of interest, analysis of mice tissues demonstrated that ARI3 cell treatment maintained the bone marrow (BM) with the lowest levels of disease in comparison with untreated mice (online supplemental figure 2C). However, mice treated with either CB-NK or ARI3 cells presented a higher percentage of tumor cells in the spleen compared with both untreated and ARI1-treated animals (online supplemental figure 2C).

In a model of low tumor burden, treatment with either CB-NK or ARI3 cells alone was not able to stop disease progression (figure 2A-2C). ARI3 cells maintained the $\mathrm{BM}$ free of disease (figure 2D), but as previously observed in the high tumor burden model (online supplemental figure 2C), a high number of NHL cells were detected in the spleen (figure 2D). The addition of exogenous IL2 to ARI3 cells was highly detrimental by causing NHL progression, as likely due to the fact that IL2 also induces $\mathrm{B}$ cell proliferation, ${ }^{23}$ with this group showing a dramatic increase in the number of NHL cells in the BM compared with ARI3 cells without exogenous IL2 and a high number of NHL cells in the spleen (figure 2D). These findings were in agreement with the role of IL2 promoting the presence of $\mathrm{B}$ cells in the $\mathrm{BM}$ and also in the spleen but to a lesser degree. ${ }^{24}$ In all cases, the high number of NHL cells in the spleen correlated with splenomegaly (figure 2E). Moreover, NK cells were detected in the spleen in all groups treated with NK cells, so the presence of tumor cells in the spleen was not due to lack of NK cell migration (figure 2F). Treatment with either ARI1 cells alone or combined with ARI3 cells did not demonstrate differences in this model of low tumor burden (figure 2A-2D) as both treatments completely prevented NHL progression. However, higher IFN $\gamma$ production was observed in the combinatorial treatment at 31 days. No increase was seen at 24 days (figure 2G). Finally, even though, in these two groups, there were low numbers of human-derived $\mathrm{T}$ cells in the $\mathrm{BM}$ and the spleen (figure $2 \mathrm{H}$ ), the presence of CAR + cells could be detected in both groups 

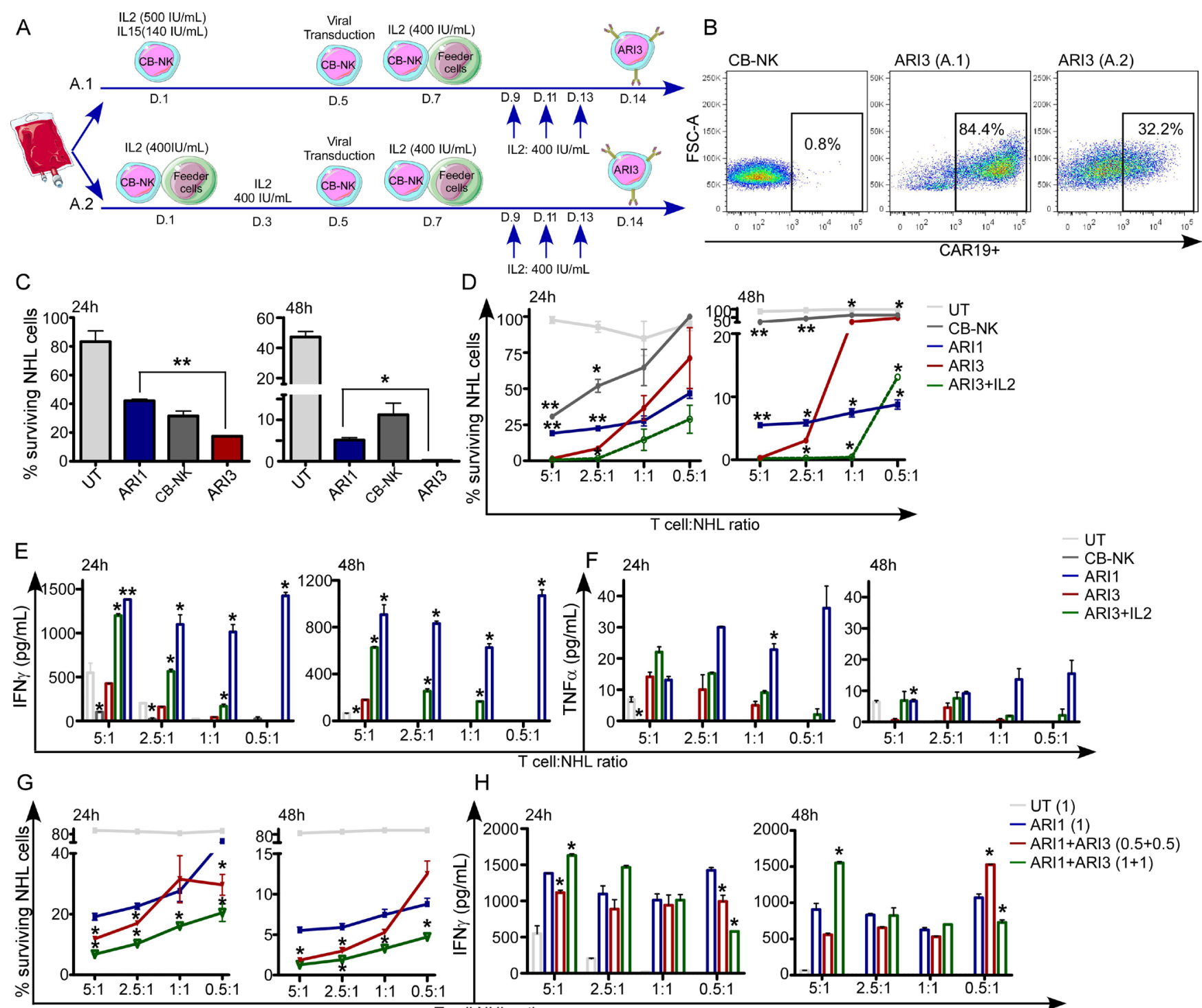

\section{$\mathrm{H}$}

-UT (1)
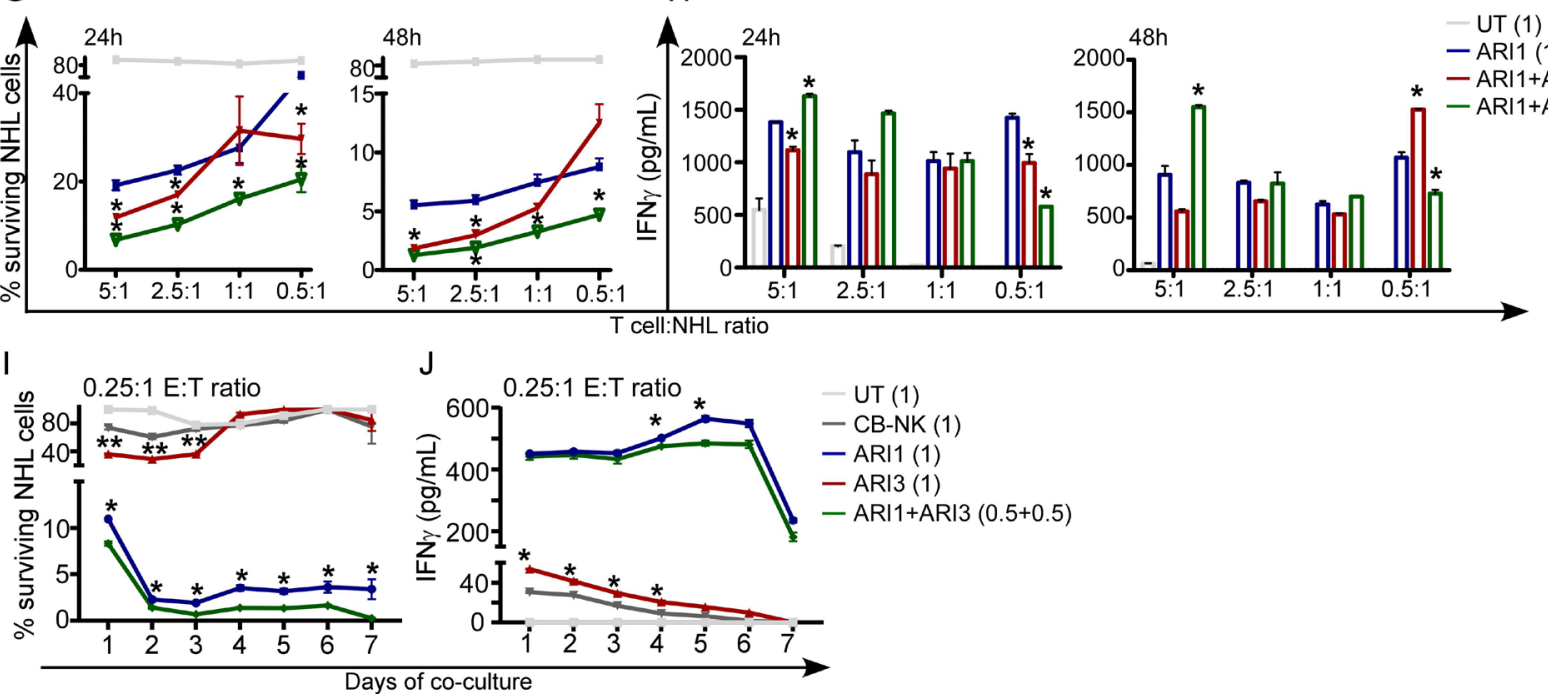

Figure 1 CAR-NK cells demonstrate higher in vitro activity than CAR-T cells only at high E:T ratios, requiring IL2 to maintain efficacy at low E:T ratios, which can be provided by CAR-T cells (see also online supplemental figure 1). (A) Diagram showing two different protocols (A.1 and A.2) used to obtain CAR-NK cells directed against CD19 (ARI3) from CB-NK. (B) Efficiencies of CB-NK transduction with both methods shown in figure part $A$. (C) Cytotoxicity assays of immune cells (untransduced T cells: UT, CAR-T cells against CD19 (ARI1), CB-NK and ARI3) against Ramos-NHL cell line performed at effector:target (E:T) ratio=10:1 at 24 hours and 48 hours. (D) Cytotoxicity assays of immune cells (UT, Ari1, CB-NK, ARI3 and ARI3 + IL2) against Ramos performed at different E:T ratios at 24 hours and 48 hours. (E) IFN $\gamma$ and (F) TNF- $\alpha$ production of the cytotoxicity assays in (D). (G) Cytotoxicity assays at 24 and 48 hours against Ramos at different E:T ratios where immune cells are added either alone UT (1), ARI1 (1), or in combination of ARI1 and ARI3 cells, where the combination could have in comparison with ARI alone, half the dose of $A R I 1$ and $A R I 3(0.5+0.5)$ or the same dose of Ari1 and ARI3 cells $(1+1)$. $(\mathrm{H})$ IFN $\gamma$ production of the cytotoxicity assays in (G). (I) Cytotoxicity assay versus Ramos cells over 7 days performed at 0.25:1 E:T ratio adding immune cells alone or the combination of $A R I 1+A R I 3(0.5+0.5)$ where the total number of immune cells is the same as in the other conditions. ( $\mathrm{J})$ IFN $\gamma$ production of the cytotoxicity assays in $(\mathrm{I})$. ${ }^{*} \mathrm{P}<0.05$. ${ }^{*} \mathrm{P}<0.0001$. Statistic in figure parts $\mathrm{D}, \mathrm{E}$ and $\mathrm{F}$ is performed comparing to $A R I 3$. Statistic in figure parts $G-J$ is performed comparing $A R I 3$ (1) or ARI1 +ARI3 (0.5+0.5) to ARI1 (1). CAR, chimeric antigen receptor; CB-NK, cord blood-derived NK cells; NK, natural killer. 
A

2Gy
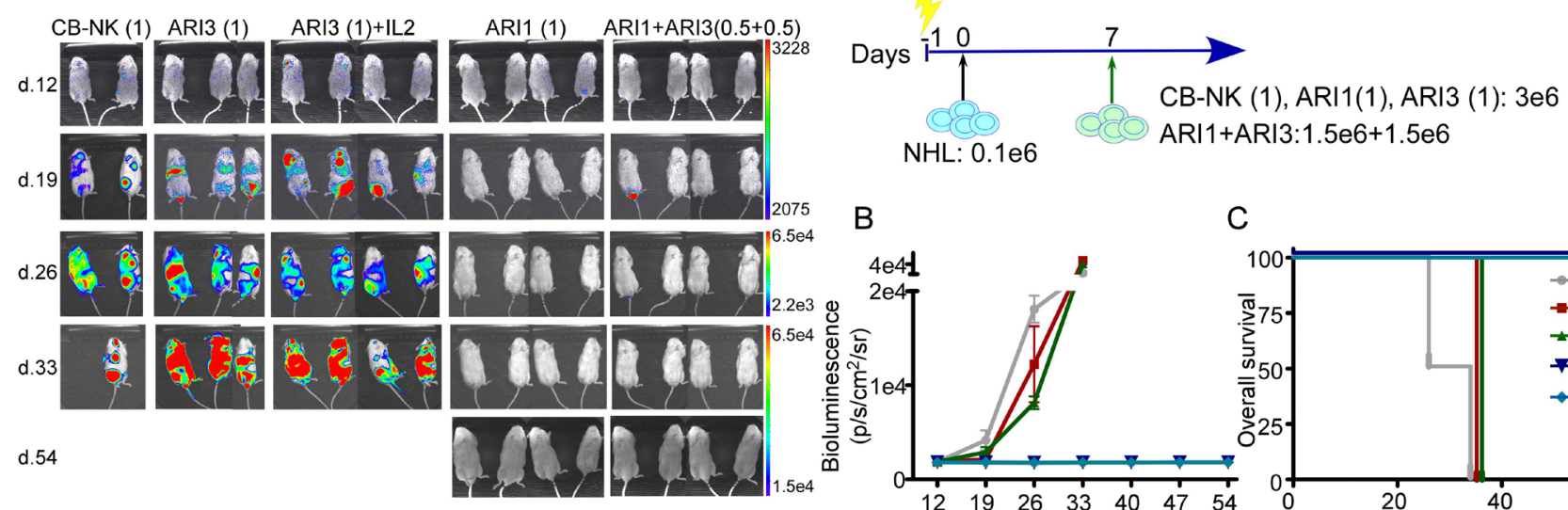

NHL: $0.1 \mathrm{e} 6$

B C
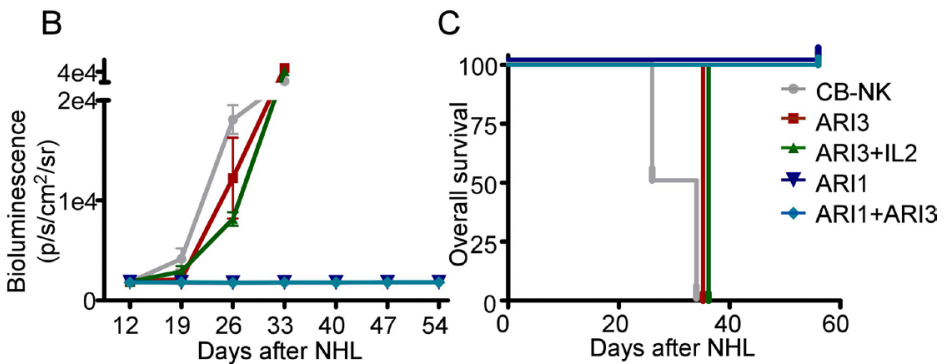

D

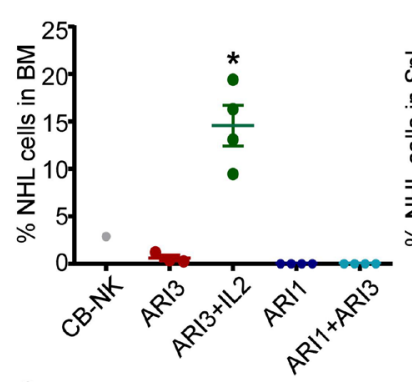

G

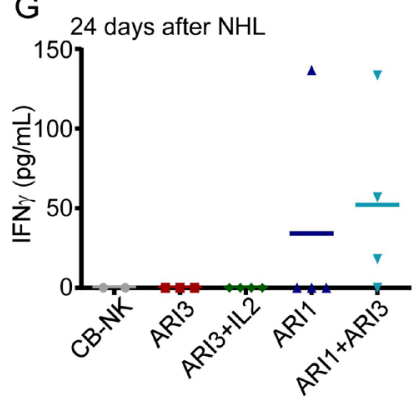

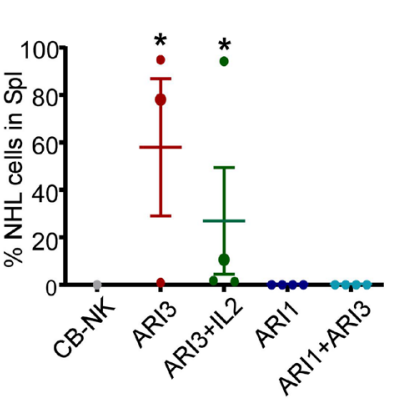

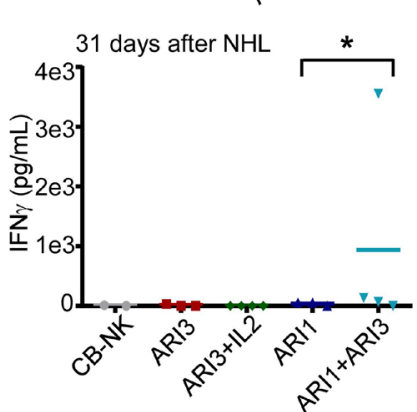

E

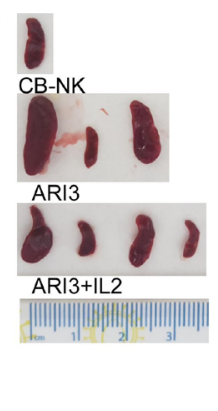

$\mathrm{H}$

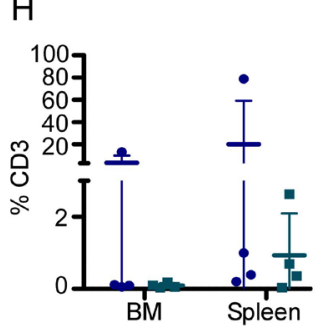

$\mathrm{F}$

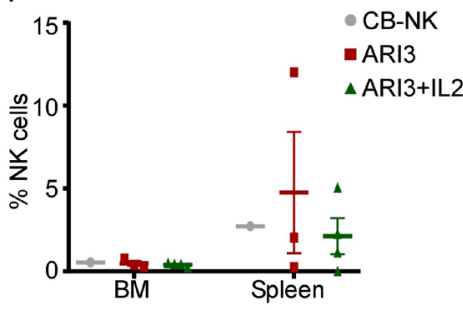

Figure 2 CAR-NK cells do not maintain in vivo efficacy and enhance the migration of tumor cells to secondary lymphoid tissues in a model of NHL. (A) In vivo efficacy in a model of low tumor burden of NHL of ARI (1) and ARI3 (1) cells alone or combined where the total number of immune cells is the same (ARI1+ARI3 $(0.5+0.5))$. The exogenous addition of IL2 was also included (ARI3 (1)+IL2) and treatment with CB-NK as control. (B) Quantification of disease progression by weekly bioluminescence imaging and $(C)$ overall survival of the different group of mice shown in figure part $A$. (D) Flow cytometry of bone marrow $(\mathrm{BM})$ and spleen of mice at the end of the experiment showing the presence of NHL cells. (E) Spleen of mice treated with NK cells (CB-NK, ARI3 and ARI3 +IL2). (F) Presence of NK cells in BM and spleen of mice treated with NK cells. (G) IFN $\gamma$ levels detected in mice serum at the time points indicated in the different groups of mice. $(\mathrm{H})$ Percentage of total $\mathrm{T}$ cells and (I) CAR-T cells in BM and spleen in mice treated with ARI1 or the combinatorial treatment based on ARI1 +ARI3 (0.5+0.5) with the same total number of immune cells. (J) Ramos cell line was used as NHL cells. ${ }^{*} \mathrm{P}<0.05$. Statistic shown in figure parts $\mathrm{D}$ and $\mathrm{G}$ is performed comparing to CB-NK. CAR, chimeric antigen receptor; CB-NK, cord blood-derived NK cells; NHL, nonHodgkin's lymphoma; NK, natural killer.

with no significant difference observed between groups (figure 2I).

\section{The number of CAR-T cells, but not CAR-NK cells, is critical in the prevention of NHL progression}

A third in vivo model of NHL with medium levels of tumor burden was performed to find out whether the combinatorial treatment based on ARI1 +ARI3 was better than ARI1 cells alone. In this model of disease, the highest number of ARI1 cells was determinant to prevent disease progression, as groups treated with ARI1 cells alone retained the disease progression for longer time than the combinatorial treatment with half dose of ARI1 cells, leading to a higher survival of the mice (figure 3A-3C). In the peripheral blood $(\mathrm{PB}), 15$ days after CAR treatment, a slight tendency for a higher production of IFN $\gamma$ was detected for the combinatorial treatment, although it was not significant, and there were no differences in the number of $\mathrm{T}$ cells present in the $\mathrm{PB}$, although it must be 
A

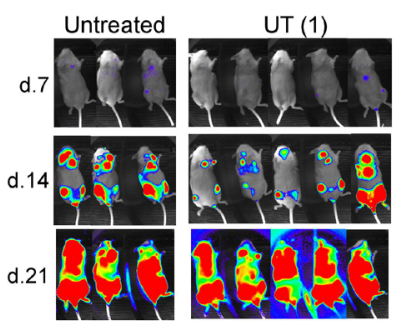

d. 28

d.32

d. 36

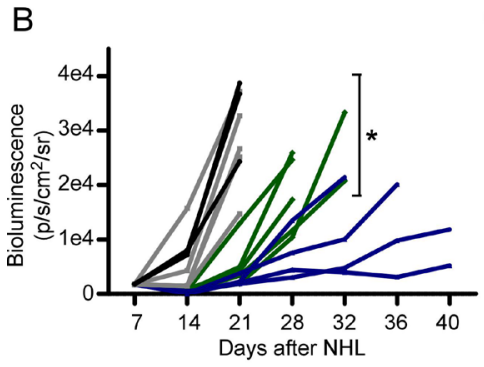

E

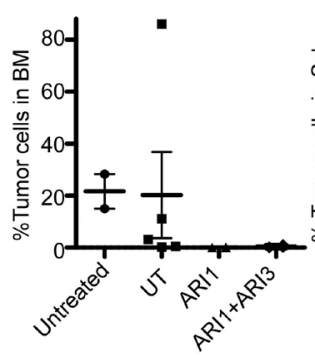

ARI1 (1)

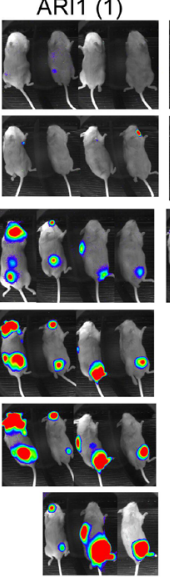

ARI1+ARI3

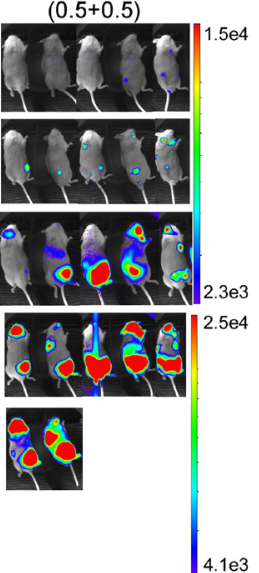

2Gy

Days

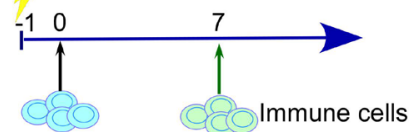

NHL: $0.35 \mathrm{e} 6 \quad$ ARI1(1): $2.7 \mathrm{e} 6$

ARI1+ARI3 $(0.5+0.5): 1.35 \mathrm{e} 6+1.35 \mathrm{e} 6$
C

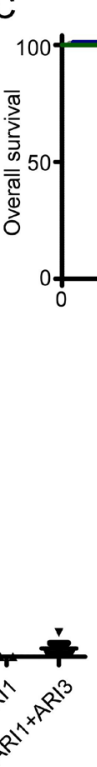

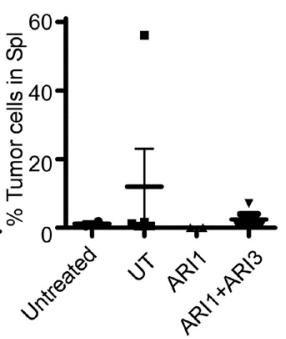
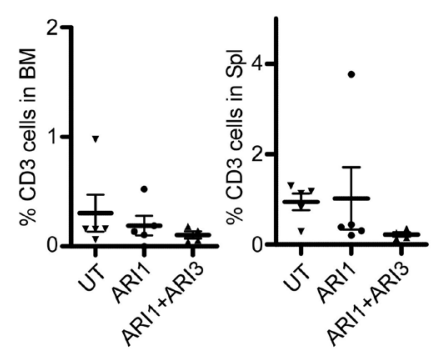

G
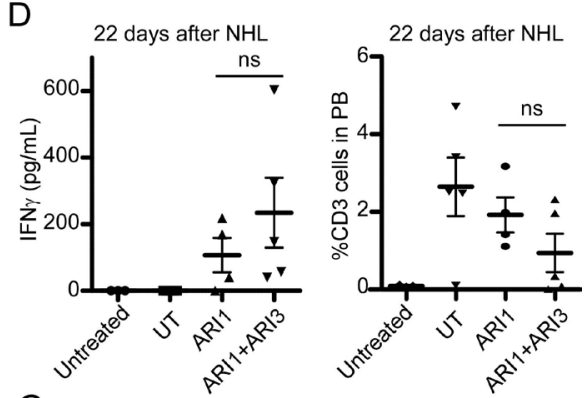

Bone Marrow

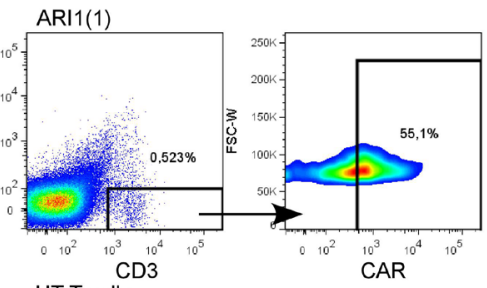

UT T cells

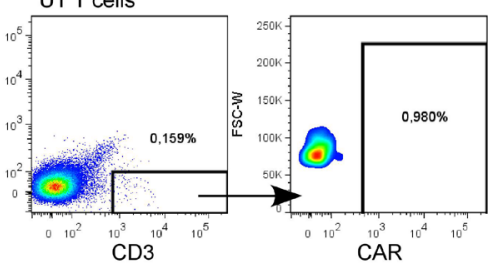



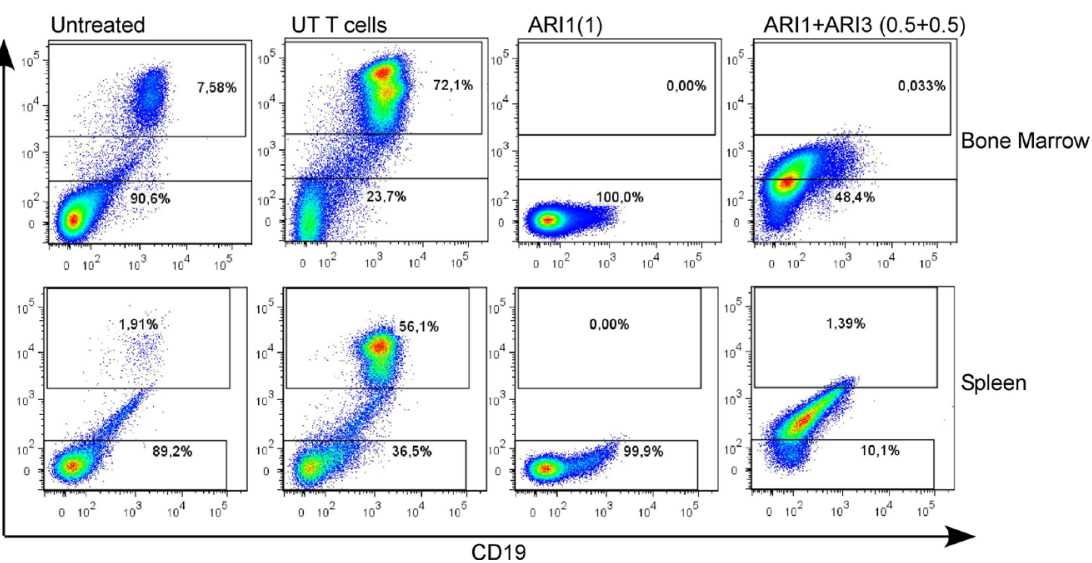

Figure 3 The number of CAR-T cells, but not CAR-NK cells, is critical in the prevention of NHL progression. (A) In vivo efficacy in a model of medium tumor burden of $\mathrm{NHL}$ of $\mathrm{ARI}$ (1) cells alone or combined with ARI3 cells where the total number of immune cells is the same (ARI1 +ARI3 (0.5+0.5)). Treatment with untransduced T cells (UT) was added as control. (B) Quantification of disease progression by weekly bioluminescence imaging and (C) overall survival of the different group of mice shown in figure part A. (D) IFN $\gamma$ levels detected in mice serum at the time points indicated in the different groups of mice. (E) Flow cytometry of bone marrow (BM) and spleen of mice at the end of the experiment showing the presence of NHL cells and (F) of T cells. (G) Flow plot showing the presence of CAR-T cells in BM in mice treated with ARI1 cells. (H) Representative flow plot showing presence of NHL cells based on GFP and CD19 expression in BM in spleen in the different groups of mice. For the group of UT T cells, the mouse with the highest number of GFP + cells is shown. Ramos cell line was used as NHL cells. ${ }^{*} \mathrm{P}<0.05$. CAR, chimeric antigen receptor; NHL, non-Hodgkin's lymphoma; NK, natural killer; NS, not significant. 
noted that the combinatorial treatment had half the dose of $\mathrm{T}$ cells (figure 3D). Analysis of mice tissues demonstrated that both treatments maintained the BM free of disease. In addition, tumor cells were not detected in the spleens of the ARI1 alone group, but a very low number of NHL cells was detected in the spleen of mice receiving the combinatorial treatment (figure 3E). In both groups, NK cells could not be detected, and the presence of T cells in both the BM and the spleen was extremely low (figure 3F), although CAR-T cells were still present (figure 3G). Of note, mice receiving the combinatorial treatment did not present a clear NHL population in the tissues. However, a population with low expression of GFP and CD19 (figure 3H), suggestive of a dying NHL cell population, was present, which might explain the higher bioluminescence signal detected in the combinatorial treatment (figure $3 \mathrm{~A}$ and $\mathrm{B}$ ).

\section{A small number of CB-NK improves the antitumor efficacy of CAR-T cells (ARI2h) in a model of MM}

Our results determined that ARI3 cells presented much lower efficacy than ARI1 cells at low E:T ratios, which translated into low in vivo efficacy (figures 1 and 2). Moreover, the combinatorial treatment based on ARI1 and ARI3 cells showed that the number of ARI1 cells and the starting tumor cell burden was determinant to prevent disease progression (figure 3). Nevertheless, we previously determined that CB-NK are able to increase the antitumor activity of UT T cells by bringing UT T cells and MM cells into close proximity. ${ }^{20}$ Thus, we hypothesized that addition of CB-NK to CAR-T cells would increase the antitumor efficacy of CAR-T cells. Of note, patients receiving CAR-T cells receive a previous lymphodepleting treatment based on fludarabine and cyclophosphamide that removes NK cells. Moreover, NK cells represent around $10 \%$ of PB leukocytes, a much lower value than for T lymphocytes. ${ }^{25}$ Thus, we decided to design a treatment that includes NK cells, specifically with half the dose of CB-NK with respect to that of CAR-T cells. This hypothesis was tested in a model of MM and humanized CAR-T cells directed against BCMA (ARI2h cells). Addition of CB-NK to ARI2h cells increased the anti-MM in vitro efficacy. Of interest, this beneficial impact was enhanced when the $\mathrm{E}: \mathrm{T}$ (ARI2h:MM(:NK)) ratios were lower $(1: 1(: 0.5))$ vs $0.5: 1(: 0.25))$ in figure 4A. Moreover, UT T cells and ARI2h cells did not augment tumor killing to the same extent as with CB-NK when they were added at the same ratios, demonstrating that the highest beneficial effect occurred with the addition of CB-NK (figure 4A), especially at earlier time points (figure 4B). Of interest, IFN $\gamma$, TNF $\alpha$ and IL2 levels in the supernatant were not increased with the addition of CB-NK, being in some cases even slightly lower (figure 4C). Specifically, IFN $\gamma$ production by either CD4 + or CD8+ T cells in these coculture assays did not increase when CB-NK were present (figure 4D). Furthermore, long-term cytotoxicity assays performed at a very low E:T ratio (1:8), confirmed the beneficial impact of CB-NK which even prevented the regrowth of MM cells (figure 4E). Of note, the pool of ARI2h cells that patients receive contains UT T cells. Therefore, we analyzed and confirmed that CB-NK also enhanced the anti-MM activity of UT T cells (figure 4F). Moreover, at low E:T ratios, the anti-MM activity of either UT or CB-NK alone was barely detectable and was much higher when both populations were combined (figure $4 \mathrm{~F}$ ).

The beneficial impact of CB-NK over ARI2h cells was confirmed in an in vivo model for MM in terms of lower disease progression and higher survival (figure $5 \mathrm{~A}-5 \mathrm{C}$ ). Despite the beneficial impact obtained with CB-NK and ARI2h cells, mice treated only with CB-NK showed a high proportion of MM cells in the BM and the spleen (figure 5D). Moreover, IFN $\gamma$ levels and total T cells in the PB of mice 28 days after treatment (figure $5 \mathrm{E}$ ), and the presence of $\mathrm{T}$ cells in the $\mathrm{BM}$ and the spleen (figure $5 \mathrm{~F}$ ), did not differ between the ARI2h and ARI2 +CB-NK groups.

\section{CB-NK promotes the activation of ARI2h cells leading to enhanced migration of ARI2h cells to MM cells}

NK cells, as part of the innate immune system, perform their activity at earlier time points than $\mathrm{T}$ cells, ${ }^{1}$ which was concordant with the highest impact of CB-NK over ARI2h cells observed at early time points (figure 4B). Therefore, we performed time-lapse in vivo imaging to visualize differences in the activity of ARI2h cells in the presence or absence of CB-NK over a period of 14 hours. As a control, UT T cells were added at the same number and in place of CB-NK. In vivo imaging showed that CB-NK accelerated the migration of ARI2h cells to MM cells, an effect that was not observed with UT T cells (online supplemental movies, figure 6A-6C). Specifically, CB-NK migrate incredibly quickly towards tumor cells (within the first $22 \mathrm{~min}$ in figure 6B) leading to enhanced formation of immune/tumor cell clusters. In turn, this promoted the migration of ARI2h cells to MM cells (figure 6C). Moreover, whereas CB-NK came into close proximity with tumor cells remarkably quickly, they started to distance from these clusters when tumor cells had already been eliminated (from $382 \mathrm{~min}$ onward in figure $6 \mathrm{~B}$ ). In parallel, movements of UT T cells added to ARI2h cells were hardly visible (online supplemental movies, figure $6 \mathrm{~B}$ ).

The enhanced migration of CAR-T cells to MM cells due to the presence of CB-NK suggested a faster activation of CAR-T cells at earlier time points. Therefore, CD69 expression, a marker of $\mathrm{T}$ cell activation, was measured demonstrating a higher activation of CD $4+T$ cells in the presence of CB-NK (figure 6D and E). Furthermore, the secretion of cytokines in these cocultures was analyzed at 6 hours demonstrating a different secretome in the presence of CB-NK (figure 6F) and that cytokines involved in T cell migration, such as CCL3 and CCL5, were increased in the presence of CB-NK (figure 6G). Of interest, cytokines related to CRS development, such as TNFo, were not increased in the presence of CB-NK (figure 6F). 

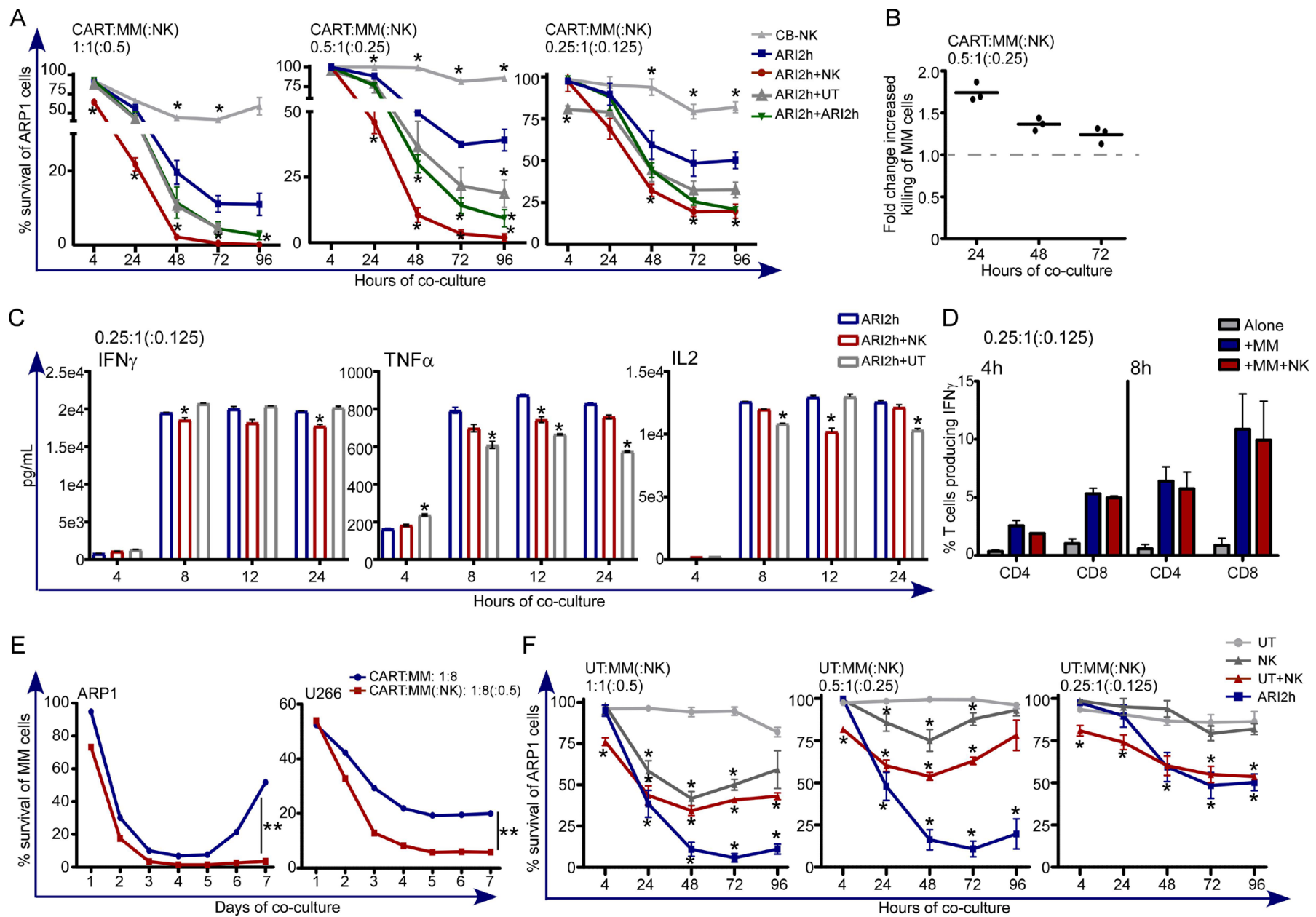

$\mathrm{F}$

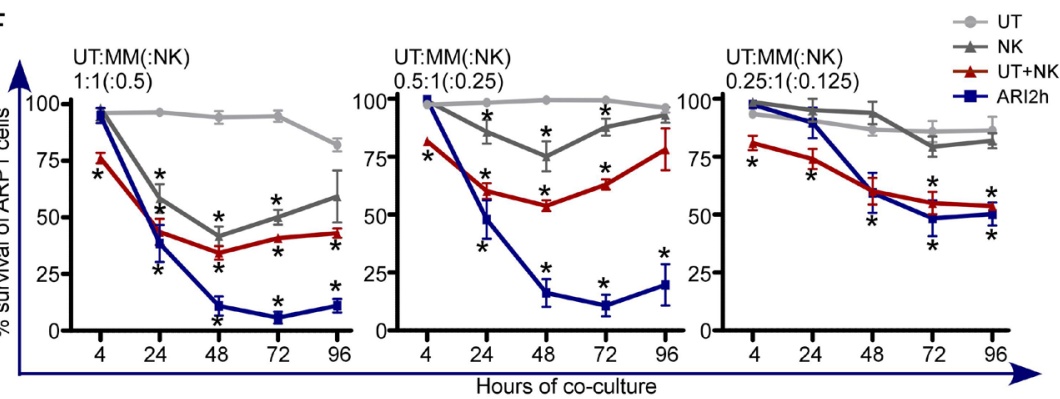

Figure 4 A small number of CB-NK improves the in vitro antitumor efficacy of CAR-T cells (ARI2h) in a model of MM (see also online supplemental figure 3). (A) Cytotoxicity assays against ARP1 (MM cell line) adding as effectors immune cells alone (CB$\mathrm{NK}$ and $\mathrm{ARI} 2 \mathrm{~h}$ ) or ARI2h cells combined with half the dose of: CB-NK (ARI2h+CB-NK), UT T cells (ARI2h+UT) or ARI2h cells (ARI2h+ARI2 hour). Assays were performed at different $E: T$ ratios. (B) Fold change increased killing with the addition of half the dose of CB-NK to ARI2h cells of cytotoxicity assay shown in figure part A at the E:T ratio of 0.5:1(:0.25) for CART:MM(:NK). (C) IFN $\gamma$, TNF $\alpha$ and IL2 production of the cytotoxicity assays in figure part A. (D) Flow cytometry analysis of IFN $\gamma$ production by $\mathrm{CD}^{+}$and $\mathrm{CD}^{+} \mathrm{T}$ cells at the time-points shown adding ARI2h:MM(:NK) at $(0.25: 1(: 0.125))$ ratio. (E) Cytotoxicity assay versus ARP1 and U266 MM cells over 7 days performed adding ARI2h:MM(:NK) at (1:8(:0.5)) ratio. In parallel, the same condition in the absence of CB-NK was performed. (F) Cytotoxicity assays against ARP1 (MM cell line) adding as effectors immune cells untransduced T (UT T) cells combined with half the dose of CB-NK (UT+CB-NK). As controls, UT T cells, NK or ARI2h cells alone were compared. Assays were performed at different (E:T) ratios. ${ }^{*} \mathrm{P}<0.05$ and ${ }^{* *} \mathrm{p}<0.0001$. Statistic in figure parts $A$ and $C$ is performed comparing to ARI2h. Statistic in figure part $\mathrm{F}$ is performed comparing UT T cells. CAR, chimeric antigen receptor; CB-NK, cord blood-derived NK cell; MM, multiple myeloma; NK, natural killer.

\section{CB-NK improves the fitness of $\mathrm{CAR}^{\text {pos }}$ and $\mathrm{CAR}^{\text {neg }} \mathrm{T}$ cells exposed to MM cells}

Last, we analyzed how the impact of CB-NK on ARI2h cells observed at early time points (figure 6) would influence ARI2h cells exposed to MM cells in long-term challenge assays. These long-term assays were performed at low E:T ratios adding cells highly diluted so that the killing would take longer (figure 7A). These experiments were performed with six different donors for $\mathrm{T}$ cells and CB-NK from three different CB units. Of interest, the impact of CB-NK over the activity of T cells was not related to differences observed in the expression of some NK cell receptors (online supplemental figure 5). We observed that after encountering MM cells, the total number of
$\mathrm{CAR}^{\text {pos }}$ and $\mathrm{CAR}^{\text {neg }} \mathrm{CD} 8+$ cells was higher in the presence of CB-NK, suggesting either a higher expansion or a lower cell death (figure $7 \mathrm{~B}$ and $\mathrm{C}$ ). The transition of memory stages of $\mathrm{T}$ cells was analyzed after production of ARI2h cells and after challenging to MM cells. In the naïve/stem cell memory (SCM) compartment, a decrease in the proportion of naïve $\mathrm{CAR}^{\text {neg }} \mathrm{CD} 4$ and $\mathrm{CD} 8 \mathrm{~T}$ cells was observed during the expansion, with no changes observed in the SCM compartment (online supplemental figure $4 \mathrm{~A}$ ).

In the central memory (CM) and effector memory (EM) compartments, at day 0 , before production of ARI2h cells, there was a lower proportion of CM in comparison with EM T cells in both $\mathrm{CAR}^{\text {pos }}$ and $\mathrm{CAR}^{\text {neg }} \mathrm{T}$ cells (figure $7 \mathrm{D}$ 
A

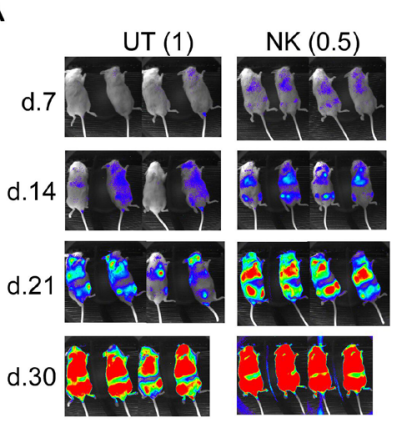

d. 37

d. 45

d. 50

d.55
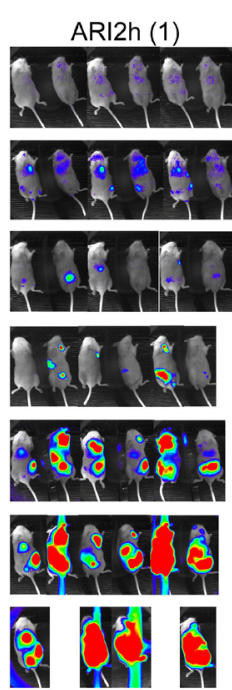

Q

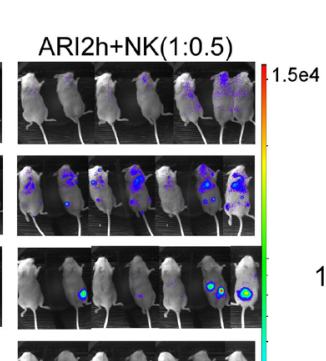

2Gy
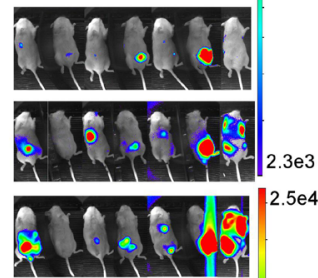

$2.5 \mathrm{e} 4$

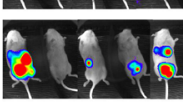

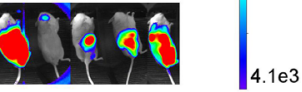

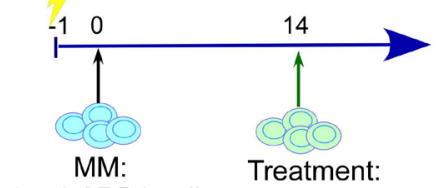

1.25e6 ARP1 cells UT: 7e6 T cells

NK (0.5): $3.5 e 6$ NK cells

ARI2h (1): 7e6 T cells (3.5e6 ARI2h)
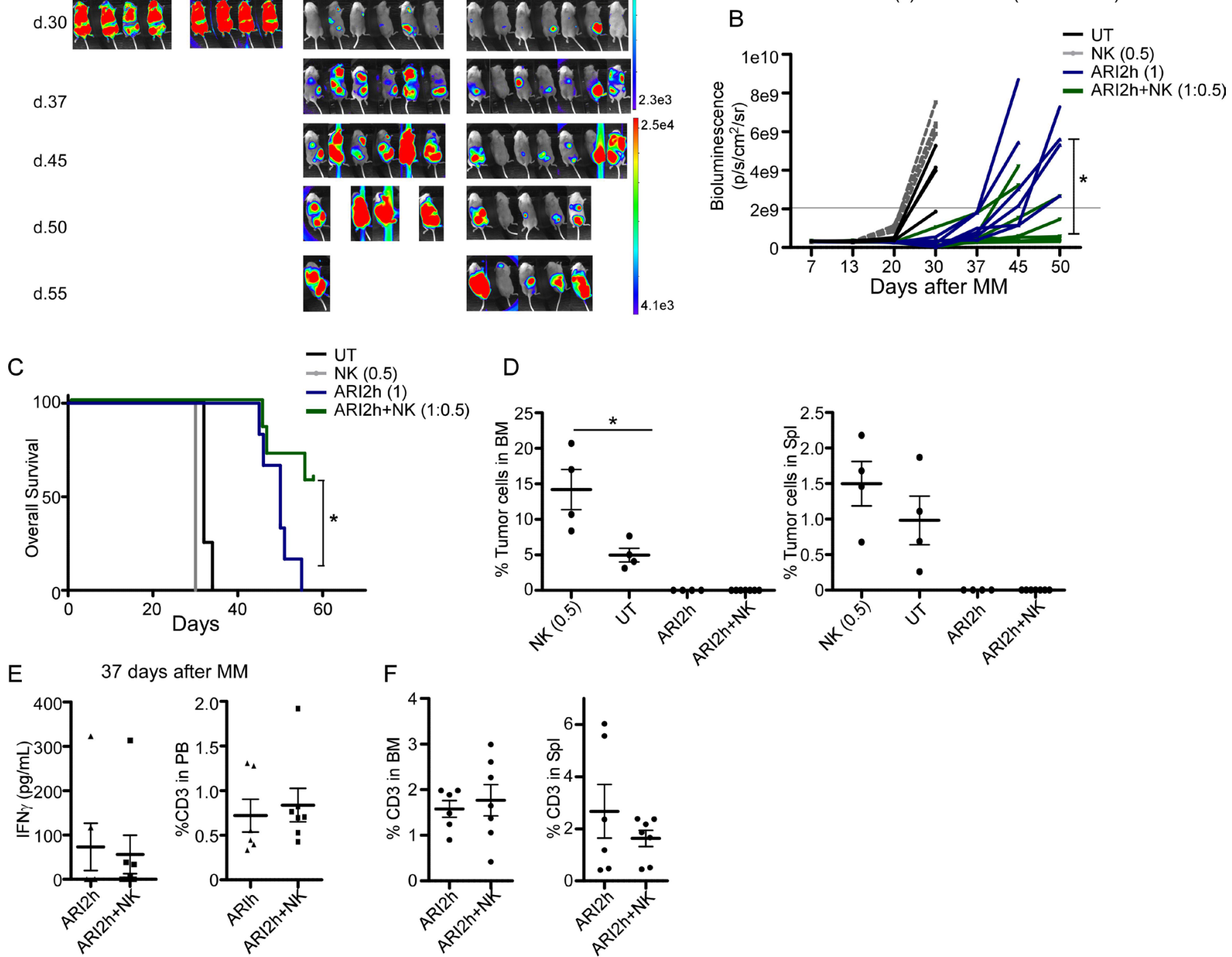

$\mathrm{F}$

D
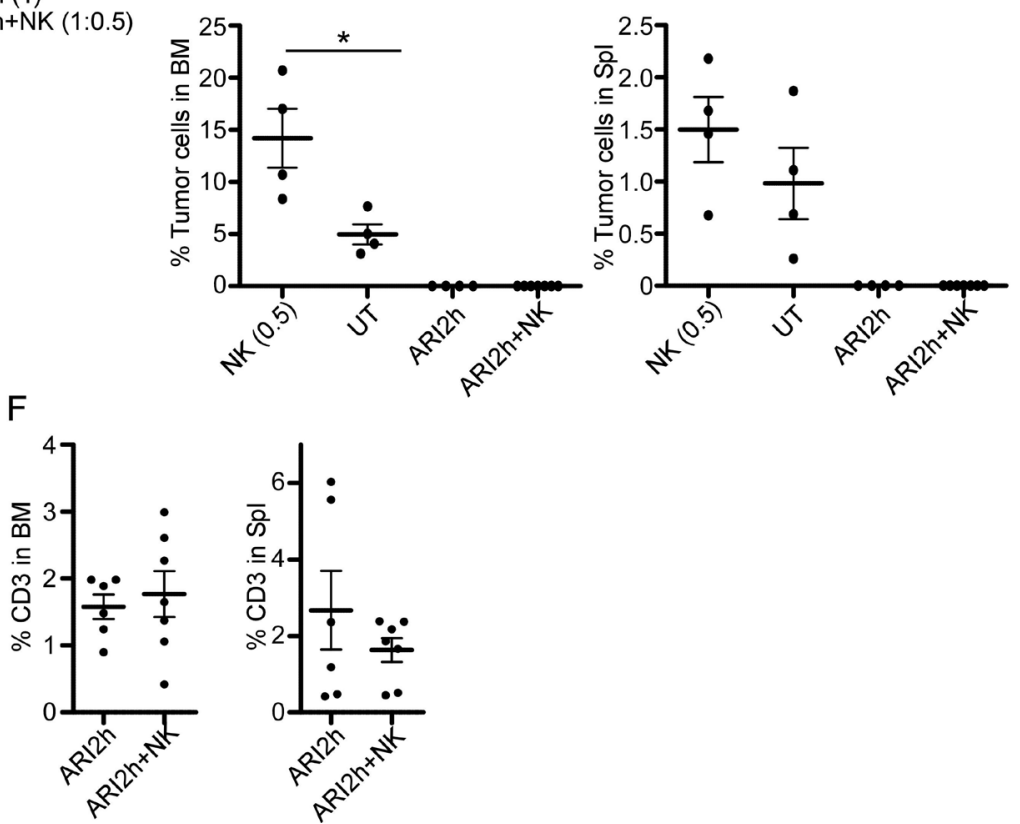

Figure 5 A small number of CB-NK improves the in vivo antitumor efficacy of CAR-T cells (ARI2h) in a model of MM. (A) In vivo efficacy in a model of MM (ARP1 cells) comparing AR2h (1) alone or with addition of half the dose of CB-NK (ARI2h+NK(1:0.5)). UT T cells (UT (1)) or CB-NK alone (NK (1)) were added as controls. (B) Quantification of disease progression by weekly bioluminescence imaging and $(C)$ overall survival of the different group of mice shown in figure part $A$. (D) Flow cytometry of bone marrow (BM) and spleen of mice at the end of the experiment showing the presence of ARP1 MM cells. (E) IFN $\gamma$ levels and CD3 T cells detected in mice serum and peripheral blood (PB) at the time point indicated in the different groups of mice. (F) Percentage of total T cells in BM and spleen in mice treated with ARI2h or ARI2h and half the dose of CB-NK (ARI2h+NK). ${ }^{*} \mathrm{P}<0.05$. CAR, chimeric antigen receptor; $\mathrm{CB}-\mathrm{NK}$, cord blood-derived natural killer cell; $\mathrm{MM}$, multiple myeloma; UT, untransduced.

and online supplemental figure 4B). Of note, CM T cells present higher capacity for homing to secondary lymphoid organs, while EM T cells are more cytolytic and express integrins and chemokine receptors necessary for localization to peripheral tissues. ${ }^{26}$ The production/expansion of ARI2h cells induced an increase in CM and a decrease in $\mathrm{EM}$ for $\mathrm{CAR}^{\mathrm{pos}} \mathrm{CD} 4$ and $\mathrm{CD} 8 \mathrm{~T}$ cells (figure 7D). For $\mathrm{CAR}^{\text {neg }} \mathrm{T}$ cells, the expansion only caused an increase in the CM compartment (online supplemental figure 4B). After exposure to MM cells, the proportion of $\mathrm{CM} \mathrm{CAR}^{\text {pos }}$ CD4 $\mathrm{T}$ cells decreased concomitant with an increase in EM $\mathrm{CAR}^{\mathrm{pos}}$ cells (figure 7D). In the presence of CB-NK, these 
A
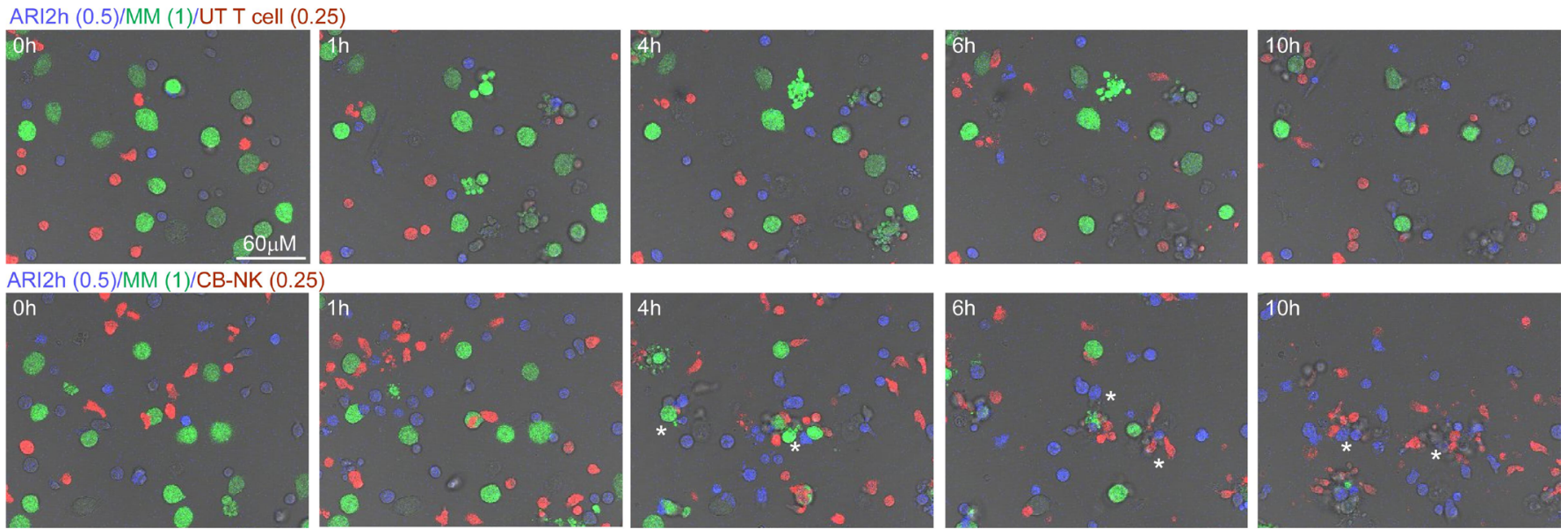

B

C

D
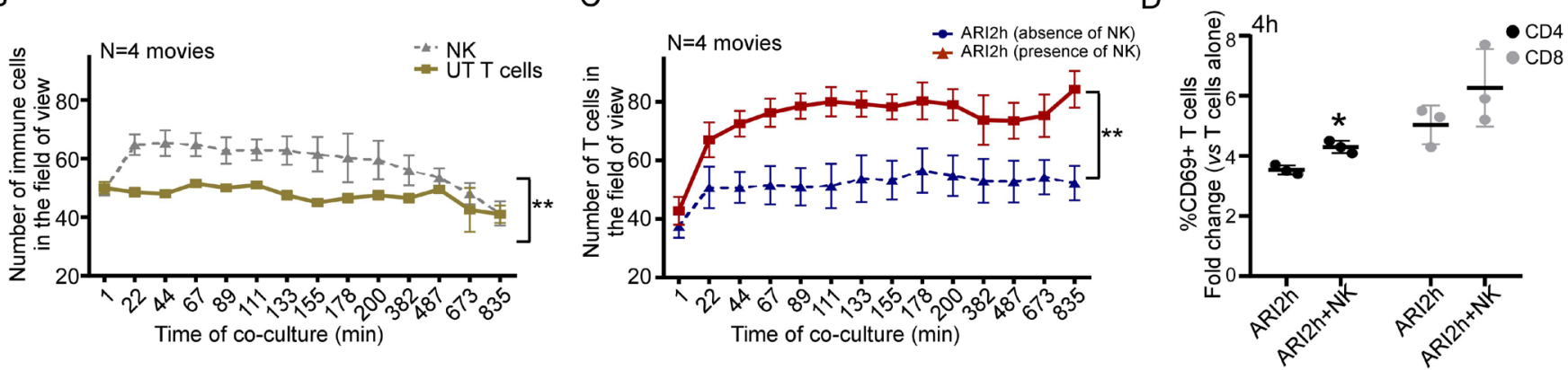

E

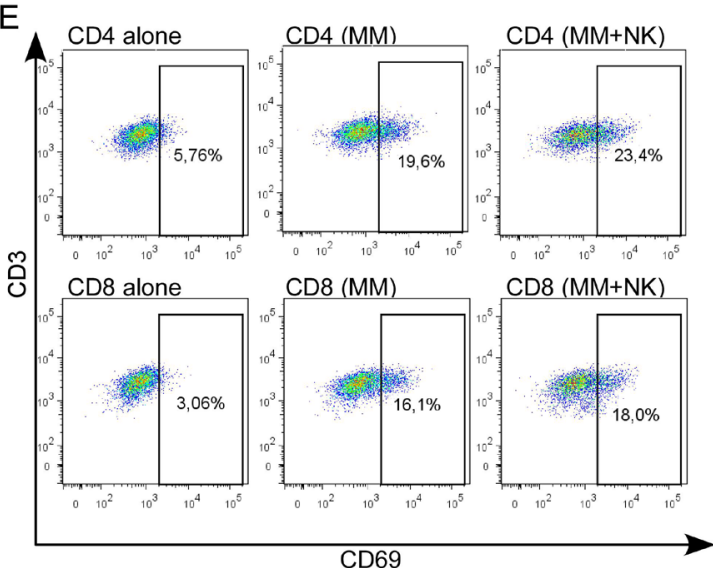

F

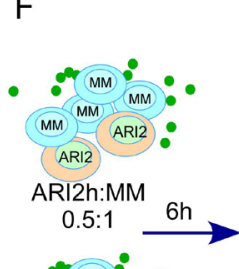

$\mathrm{N}=4$

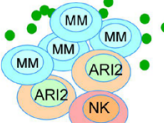

ARI2h:MM:NK

0.5:1:0.25

Figure 6 CB-NK promotes the activation of ARI2h cells leading to enhanced migration of ARI2h cells to MM cells (see also online supplemental movies 1 and 2). (A) Time lapse images over 14 hours of ARI2m cells cocultured with RPMI8226 MM cells modified to express green fluorescent protein and adding CB-NK at half the dose of ARI2h cells. In parallel, as control, UT T cells were added at half the dose of ARI2h cells. ARI2h and MM cells were added at 0.5:1 E:T ratio. ARI2h cells were stained with cell tracker CMAC and UT T cells or CB-NK with cell tracker Deep Red. (B) Analysis of the number of CB-NK or UT T cells and (C) Of ARI2h cells in the presence/absence of CB-NK in each field of view at the different time-points shown for experiment shown in figure part A). Data are calculated with four different time lapse images over 14 hours each. Movies with CB-NK derived from different CB units demonstrated the same pattern. (D) Percentage of CD69 ${ }^{+}$cells in the CD4 ${ }^{+}$and CD8 $^{+}$ $\mathrm{ARI} 2 \mathrm{~h}^{+}$cells in the presence (ARI2h+NK) or absence (ARI2h) of CB-NK after coculturing ARP1 MM cells with ARI2h cells. (E) Representative plot of analysis shown in figure part D. (F) ARP1 MM cells were co-cultured with ARI2h cells for 6 hours at $0.5: 1$ $(\mathrm{E}: \mathrm{T})$ ratio in the presence or absence of half the dose of CB-NK. Supernatants were taken and analyzed using a ProcartaPlex multiplex immunoassay kit $(n=4)$. Heat map shows the relative abundance of each molecule on a scale of blue (low) to red (high). (G) Molecules significantly differentially expressed in the two groups of heat map shown in figure part $\mathrm{F}$. ${ }^{*} \mathrm{P}<0.05$ and ${ }^{\star \star} \mathrm{p}<0.0001$. CB-NK, cord blood-derived NK cell; E:T, effector:target; MM, multiple myeloma; UT, untransduced.

changes were more pronounced (figure 7D), suggesting a more differentiated phenotype and a faster capacity of CAR-T cells to migrate where MM cells are emerging.
For $\mathrm{CAR}^{\mathrm{pos}} \mathrm{CD} 8 \mathrm{~T}$ cells, exposure to MM cells caused an increase in the EM compartment only in the presence of CB-NK (figure 7D). Of interest, no changes were observed 
A
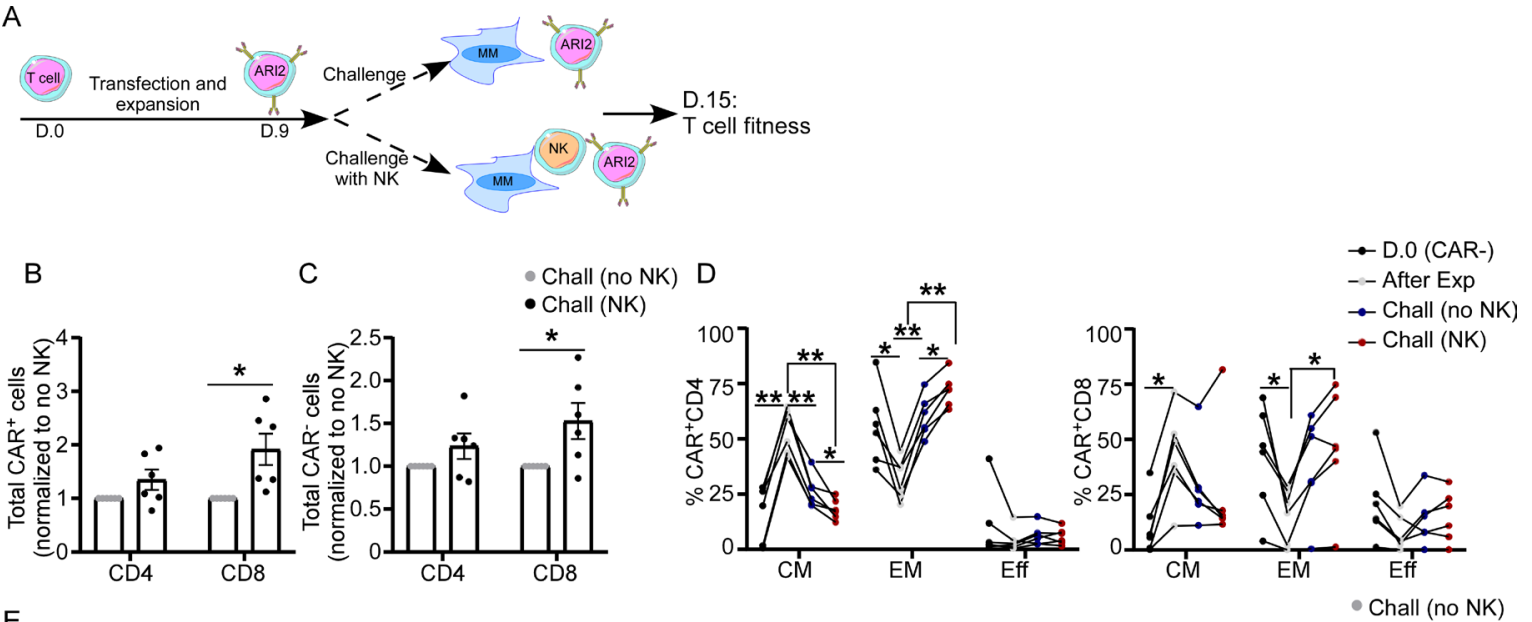

E $\quad \mathrm{CAR}^{+} \mathrm{CD4}^{+}$

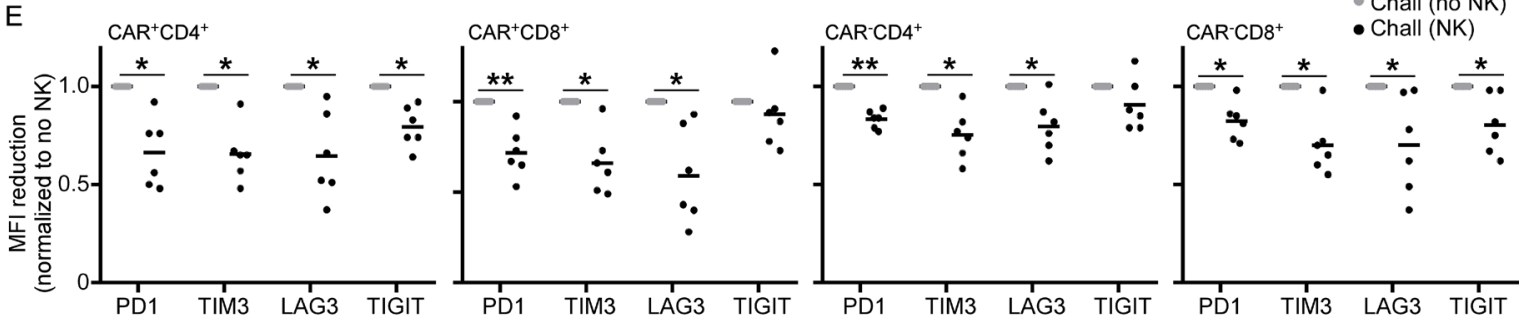

$\mathrm{F}$
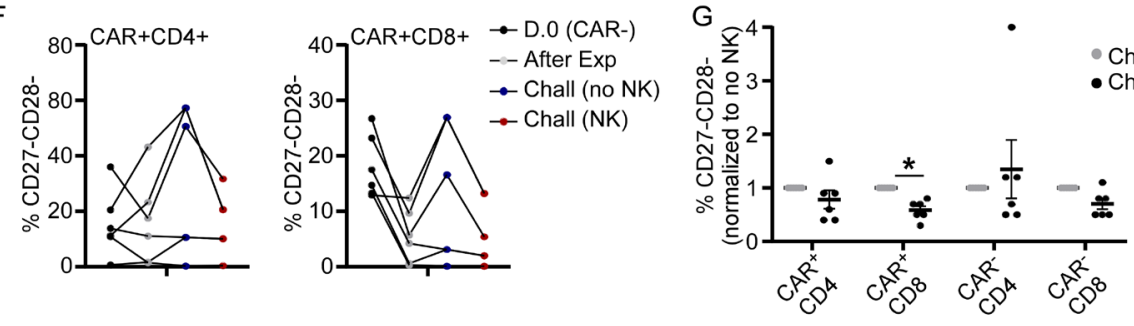

Chall (no NK)

- Chall (NK)

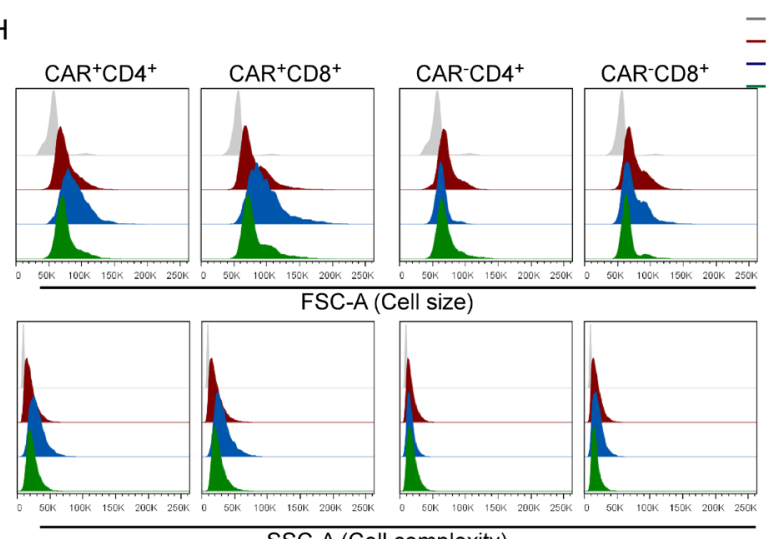

SSC-A (Cell complexity)

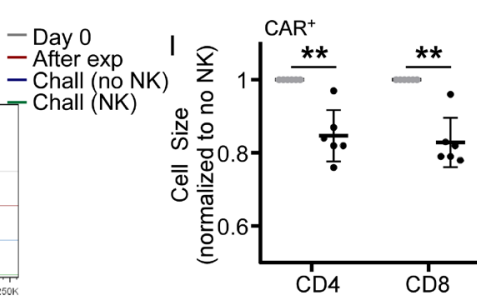

- Chall (no NK)
- Chall (NK)
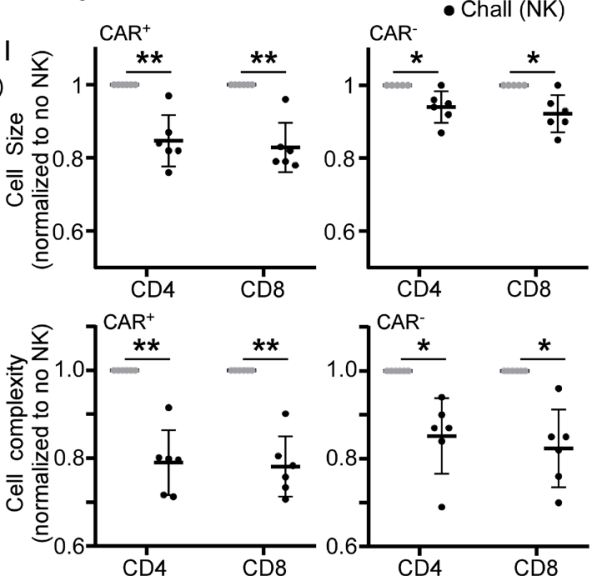

Figure 7 CB-NK improves the fitness of $C A R^{\text {pos }}$ and $C A R^{\text {neg }} T$ cells exposed to MM cells (see also online supplemental figure 4 and 5). (A) T cells (D.0) were expanded and transduced to produce ARI2h cells (after Exp), which were cocultured in vitro for 6 days with ARP1 MM cells at 0.5:1 (E:T) ratio in the presence (Chall (NK)) or absence of CB-NK (Chall (no NK)). \% of ARI2h cells was normalized at $40 \%$ for all assays. At those time points, the fitness of $T$ cells was analyzed by flow cytometry (B-l). (B-C) Variation of the total number of $\mathrm{CAR}^{\text {pos }}(\mathrm{B})$ and $\mathrm{CAR}{ }^{\text {neg }}(\mathrm{C}) \mathrm{CD}^{+}$and $\mathrm{CD} 8^{+}$cells after challenge to MM cells in the presence or absence of CB-NK. (D) Memory phenotype (CM: central memory; EM: effector memory; Eff: effector) of CD4 ${ }^{+}$and CD8 ${ }^{+}$ARI2h ${ }^{+}$ cells at the different time points shown in figure part $\mathrm{A}$. (E) Analysis of exhaustion markers of $\mathrm{CD} 4^{+}$and $\mathrm{CD} 8^{+} \mathrm{ARI} 2 \mathrm{~h}^{+}$and $\mathrm{ARI} 2 \mathrm{~h}$ cells after challenge to Arp1 cells in the presence/absence of CB-NK. (F-G) \% of CD27-CD28 ${ }^{-}$cells in $\mathrm{CD}^{+}$and $\mathrm{CD} 8^{+} \mathrm{ARI} 2 \mathrm{~h}^{+}$ and $\mathrm{ARI} 2 \mathrm{~h}^{-}$cells at the different time points shown in figure part A. Figure part $\mathrm{F}$ is a representative plot of variation levels at the different time points, and figure part $\mathrm{G}$ is the comparative analysis versus the presence/absence of $\mathrm{CB}-\mathrm{NK}$. (H and I) Cell size and complexity of $\mathrm{CD}^{+}$and $\mathrm{CD}^{+} \mathrm{ARI} 2 \mathrm{~h}^{+}$and $\mathrm{ARI} 2 \mathrm{~h}^{-}$cells at the different time points shown in figure part $\mathrm{A}$. Plots shown at day 0 in figure parts $D$ and $F$ correspond to $A R I 2 h^{-}$cells (before transduction). After Exp: after production of ARI2h cells. Experiments were performed with six donors of T cells and CB-NK from three CB units. Phenotype of CB units is shown in online supplemental figure 5. ${ }^{*} \mathrm{P}<0.05$ and ${ }^{* *} \mathrm{p}<0.0001$. CB-NK, cord blood-derived NK cells; Chall, challenge of immune cells to MM cells; E:T, effector:target; MM, multiple myeloma; NK, natural killer. 
in the number of terminally differentiated effector ARI2h cells (figure 7D). However, an increase in effector cells was noticed for both $\mathrm{CAR}^{\text {neg }} \mathrm{CD} 4+$ and $\mathrm{CD} 8+\mathrm{T}$ cells that was more pronounced for $\mathrm{CD} 8+\mathrm{T}$ cells in the presence of CB-NK (online supplemental figure 4B).

Moreover, the fitness of ARI2h cells after exposure to MM was analyzed by looking at exhaustion and immunosenescent parameters. The presence of CB-NK decreased the expression of exhaustion markers PD-1, TIM3 and LAG3 in both $\mathrm{CAR}^{\text {pos }}$ and $\mathrm{CAR}^{\text {neg }} \mathrm{T}$ cells. Furthermore, TIGIT levels were also reduced in $\mathrm{CAR}^{\mathrm{pos}} \mathrm{CD} 4+\mathrm{T}$ cells and both $\mathrm{CAR}^{\text {neg }} \mathrm{CD} 4+$ and CD8 $+\mathrm{T}$ cells (figure $7 \mathrm{E}$ ).

Loss of CD27 and CD28, known as markers related to the development of immunosenescence, specifically after chronic viral infection, ${ }^{27-29}$ were also improved in the presence of CB-NK. Specifically, exposure to MM cells induced an increase in the emergence of $\mathrm{CD} 27^{-} \mathrm{CD} 28^{-}$ cells in $\mathrm{CAR}^{\text {pos }} \mathrm{T}$ cells (figure $7 \mathrm{~F}$ ), a detrimental impact that was reduced in the presence of CB-NK for the $\mathrm{CAR}^{\text {pos }} \mathrm{CD} 8+\mathrm{T}$ cell population (figure $7 \mathrm{G}$ ). Senescent cells are also characterized by presenting a higher cell size, an event that impairs cell proliferation. ${ }^{30}$ Importantly, production/expansion of ARI2h cells induced an increase in both cell size and complexity for all $\mathrm{CAR}^{\text {pos }}$ and $\mathrm{CAR}^{\text {neg }} \mathrm{T}$ cells that continued to rise after exposure to MM cells (figure $7 \mathrm{H}$ ). CB-NK avoided the increase in cell size and cell complexity of both $\mathrm{CAR}^{\text {pos }}$ and $\mathrm{CAR}^{\text {neg }} \mathrm{CD}^{+}$ and $\mathrm{CD}^{+} \mathrm{T}$ cells after exposure to MM cells (figure $7 \mathrm{I}$ ) suggesting a delayed emergence of senescence in ARI2h cells due to the presence of CB-NK.

\section{DISCUSSION}

CAR-T cell immunotherapy has modified the treatment of hematological malignancies. However, optimal clinical results fluctuate depending on the disease. Specifically, pediatric ALL patients treated with CART-19 cells maintain durable responses with the presence of CAR-T cells many years after administration, ${ }^{3}$ whereas older patients with conditions such as $\mathrm{NHL}^{10}$ and $\mathrm{MM}^{16}$ present poorer responses due to lack of persistence of CAR-T cells. This lack of persistence, among other factors, might be due to a decreased fitness of the immune cells acquired with aging. ${ }^{29}{ }^{31}$ Here, we demonstrate that CB-NK can be combined with CAR-T cells, leading to enhanced in vivo antitumor efficacy without increasing parameters related to CRS in a model of MM. Importantly, CB-NK enhanced the fitness of CAR-T cells after in vitro exposure to MM cells promoting lower levels of exhaustion and senescence, suggesting the use of CB-NK as an approach to enhance the efficacy of CAR-T cell treatment.

A lot of strategies are being explored to enhance the efficacy of CAR-T cells without increasing associated CRS and neurotoxicity. These possibilities include the creation of complex constructs to enhance the persistence of CAR-T cells ${ }^{32}$ and also the use of universal allogeneic CAR-T cells that are derived from more 'healthy' $T$ cells. In this regard, cord blood units can be used as a source to obtain 'off-the-shelf' immune cells including CB-NK and CAR-NK cells. ${ }^{16} 1733$ Clinical studies indicate that NK cells are mostly effective as a consolidation therapy in AML patients, ${ }^{74-36}$ but their efficacy is much lower for other malignancies. ${ }^{7}$ However, CAR-NK cells derived from CB-NK have demonstrated safety in NHL patients. However, the durability of response or CAR NK persistence is unknown, ${ }^{637}$ suggesting that improvements either in the CAR construct or changes in clinical protocols might still be needed to ensure long-term responses. Here, we confirm that in comparison with CAR-T cells, CAR-NK cells are only effective at high doses (high E:T ratio), that their activity decreases rapidly over time, and that, in addition, they require IL2, which can be provided by $\mathrm{T}$ cells. In addition, the combination of CAR-NK and CAR-T cells demonstrated that a minimum number of CAR-T cells is required to achieve responses, an event confirmed in clinical studies. ${ }^{38}$ An ideal immune cell treatment should be able to maintain efficacy at the lowest dose possible and to persist long enough to avoid relapses. These results added to our previous findings demonstrating that proteins secreted by CB-NK enhance the anti-MM activity of UT T cells ${ }^{20}$ led us to hypothesize and confirm that CB-NK could be a tool to enhance the efficacy of CAR-T cells.

The choice of adding half the dose of CB-NK versus CAR-T cells was based on clinical studies in cancer where infusion of very high and repetitive doses of NK cells has not shown efficacy. ${ }^{39}$ Moreover, studies in microbial infections show that NK cells and T cells kill each other to avoid detrimental consequences of excessive inflammation. Thus, in cases of massive viral and bacterial infections, NK cells kill $\mathrm{T}$ cells to avoid a devastating inflammatory response by $\mathrm{T}$ cells that could lead to death. ${ }^{40-44}$ Similarly, regulatory T (T-reg) cells eliminate NK cells to avoid an exacerbated inflammatory response mediated by NK cells, ${ }^{45}$ suggesting the relevance of maintaining an optimal ratio between both populations. This intricate regulation occurs also in patients with cancer receiving NK cells, where responding patients had lower levels of T-reg cells than non-responding patients, ${ }^{46}$ and where T-reg depletion led to improved responses. ${ }^{35}$ However, another study demonstrated increased T-reg cells in responding patient ${ }^{47}$ indicating the complexity of these interactions. This complex regulation between both cell populations added to the fact that PB NK cells are present in much lower numbers than $\mathrm{T}$ cells led us to use only a half dose of CB-NK in comparison with CAR-T cells.

The innate antitumor activity of NK cells occurs at earlier time points than that of adaptive T cells, where NK cells are important in the regulation of the activity of adaptive T cells. ${ }^{48}$ We demonstrate that both CAR-NK cells and CB-NK initiate their antitumor activity earlier than CAR-T cells, which significantly, in the case of CB-NK, led to a faster activation of CAR-T cells and promotion of their migration to MM cells at early time points. Of note, prior to receiving CAR-T cells, patients undergo a lymphodepleting conditioning regimen ${ }^{15} 16$ to eliminate residual 
$\mathrm{T}$ cells and thus avoid a possible immunogenicity against murine components of the CAR. ${ }^{31}$ This conditioning regimen also depletes NK cells for at least 15 days, ${ }^{49}$ a time at which the performance of CAR-T cells is crucial. Our results show that adding a low number of CB-NK in the CAR product led to an enhancement of the efficacy of CAR-T cells after the first days of CAR-T cell treatment with no enhancement in the CRS and improved fitness of CAR-T cells. In addition, the immunoregulatory role of NK cells in avoiding detrimental inflammation by $\mathrm{T}$ cells, as observed in viral infections, ${ }^{40}$ could represent an advantage here to avoid severe CRS during the initial period after administering CAR-T cells.

Moreover, CB-NK enhanced the health of both $\mathrm{CAR}^{\text {neg }}$ and $\mathrm{CAR}^{\text {pos }} \mathrm{T}$ cells, with lower expression of exhaustion and immunosenescence markers after exposure to $\mathrm{MM}$ cells. Exhausted or senescent $\mathrm{T}$ cells negatively impact the long-term persistence of CAR-T cells, ${ }^{50} 51$ which is critical to avoid relapses. ${ }^{31516}$ T cell exhaustion is a common problem in cancer, which can be reversed with immunecheckpoints inhibitors. Specifically, MM patients receiving an autologous stem cell transplant develop exhausted CD4 and senescent CD8 T cells, with high expression of LAG3 and TIM3 associated with poor outcomes. ${ }^{52}$ Moreover, T cell immunoreceptor with Ig and ITIM domains (TIGIT) is another potential target of immune modulation in MM patients. ${ }^{53}$ Given the fact that clinical trials with immunocheckpoint inhibitors in MM were halted due to their high toxicity, ${ }^{54}$ the impact of CB-NK ameliorating exhaustion in T cells could represent a promising alternative. Moreover, MM patients are an elderly population with senescent $\mathrm{T}$ cells with lower proliferative capacity and impaired function. ${ }^{55}$ Here, CB-NK reversed parameters related to senescence in both $\mathrm{CAR}^{\text {neg }}$ and $\mathrm{CAR}^{\text {pos }} \mathrm{T}$ cells, suggesting an interesting approach to improve immunosenescence in $\mathrm{T}$ cells in MM.

In summary, CB-NK and CAR-NK cells can be used as universal sources of immune cells for the treatment of cancer patients. However, their lower efficacy in comparison with CAR-T cells highlights the need to look for novel strategies to improve their therapeutic potential. Here, we present CB-NK as a novel approach to enhance the efficacy of CAR-T cells in a model of MM, importantly without enhancing parameters related to CRS development. Moreover, CB-NK prevent the emergence of exhausted and senescent $\mathrm{T}$ cells after exposure to MM cells, which suggests they could lead to enhanced persistence of CAR-T cells.

\footnotetext{
Author affiliations

${ }^{1}$ Department of Hematology, Hospital Clinic, IDIBAPS, Barcelona, Spain

${ }^{2}$ Department of Pediatrics-Research, The University of Texas MD Anderson Cancer Center, Houston, Texas, USA

${ }^{3}$ CIRI, Université de Lyon, INSERM U1111, ENS de Lyon, Université Lyon 1, Lyon, France

${ }^{4}$ Université Côte d'Azur, INSERM, Nice, France

${ }^{5}$ Department of Immunology, Hospital Clinic de Barcelona (HCB), Platforms of Immunoterapy IDIBAPS HSJD-HCB and BST-HCB, Barcelona, Spain
}

${ }^{6}$ Department of Medicine, University of Barcelona, Barcelona, Catalunya, Spain ${ }^{7}$ Department of Hematology. University of Barcelona, IDIBAPS, Barcelona, Spain ${ }^{8}$ Josep Carreras Leukaemia Research Institute, Barcelona, Spain

${ }^{9}$ Department of Experimental Hematology, Instituto de Investigación SanitariaFundación Jiménez Diaz, Madrid, Spain

Acknowledgements We would like to acknowle Multiple Myeloma Research Center (Little Rock, Arkansas) for providing the ARP1 cell line. We would also like to acknowledge Dean A Lee (The Ohio State University School of Medicine) for providing the feeder cells. We would like to acknowledge the facility of Confocal Fluorescence Microscopy at the University of Barcelona.

Contributors BM-A conceived and designed the study, LP-A performed in vitro and in vivo experiments related to chimeric antigen receptor (CAR)-natural killer (NK) cells and non-Hodgkin's lymphoma (NHL). MB performed in vitro and in vivo experiments related to the combination of cord blood-derived NK (CB-NK) with CAR-T cells in multiple myeloma. LP-A, MB and BM-A analyzed data and wrote the manuscript. EV provided the BaEV plasmid. AN designed the scFv of ARI2 and provided plasmid coding for GFP-Ffluc. AMB performed the multiplex immunoassays, designed the flow cytometry panels and edited the manuscript. MJ provided the construct for ARI1 and provided funding. AU-I provided funding. All authors read and approved the manuscript.

Funding La Caixa Foundation (CP042702) and Institute of Health Carlos III (projects: Pl17/01043, PI20/00991 and PI18/00775) provided funding for all studies.

Competing interests None.

Patient consent for publication Not required.

Ethics approval The study was approved by the Ethics Committee of Hospital Clinic of Barcelona (2017/0438 and 2020/0048). All animal work was performed under approval of Ethical Committee of Animal Research (University of Barcelona, procedure 230/19). Mice were euthanized when they reached a score that determined the lack of mobility, lack of response to stimuli or weight loss as specified in our protocol approved by the Ethical Committee of Animal Research of the (procedure 230/19).

Provenance and peer review Not commissioned; externally peer reviewed.

Data availability statement Data are available on reasonable request. All data relevant to the study are included in the article or uploaded as supplementary information. All information is in the manuscript. All information is in the manuscript.

Supplemental material This content has been supplied by the author(s). It has not been vetted by BMJ Publishing Group Limited (BMJ) and may not have been peer-reviewed. Any opinions or recommendations discussed are solely those of the author(s) and are not endorsed by BMJ. BMJ disclaims all liability and responsibility arising from any reliance placed on the content. Where the content includes any translated material, BMJ does not warrant the accuracy and reliability of the translations (including but not limited to local regulations, clinical guidelines, terminology, drug names and drug dosages), and is not responsible for any error and/or omissions arising from translation and adaptation or otherwise.

Open access This is an open access article distributed in accordance with the Creative Commons Attribution Non Commercial (CC BY-NC 4.0) license, which permits others to distribute, remix, adapt, build upon this work non-commercially, and license their derivative works on different terms, provided the original work is properly cited, appropriate credit is given, any changes made indicated, and the use is non-commercial. See http://creativecommons.org/licenses/by-nc/4.0/.

\section{ORCID iDs}

Anthony Matthew Battram http://orcid.org/0000-0002-9179-0639

Manel Juan http://orcid.org/0000-0002-3064-1648

Beatriz Martin-Antonio http://orcid.org/0000-0003-0612-2693

\section{REFERENCES}

1 Bachiller M, Battram AM, Perez-Amill L, et al. Natural killer cells in immunotherapy: are we nearly there? Cancers 2020;12:3139.

2 Ortíz-Maldonado V, Rives S, Castellà M, et al. CART19-BE-01: A Multicenter Trial of ARI-0001 Cell Therapy in Patients with CD19 ${ }^{+}$ Relapsed/Refractory Malignancies. Mol Ther 2021;29:636-44.

3 Maude SL, Laetsch TW, Buechner J, et al. Tisagenlecleucel in children and young adults with B-cell lymphoblastic leukemia. N Engl J Med 2018;378:439-48. 
4 Li Y, Hermanson DL, Moriarity BS, et al. Human iPSC-derived natural killer cells engineered with chimeric antigen receptors enhance antitumor activity. Cell Stem Cell 2018;23:181-92.

5 Liu E, Tong Y, Dotti G, et al. Cord blood NK cells engineered to express IL-15 and a CD19-targeted CAR show longterm persistence and potent antitumor activity. Leukemia 2018;32:520-31.

6 Liu E, Marin D, Banerjee P, et al. Use of CAR-Transduced natural killer cells in CD19-Positive lymphoid tumors. N Engl J Med 2020;382:545-53.

7 Martín-Antonio B, Suñe G, Perez-Amill L, et al. Natural killer cells: angels and Devils for immunotherapy. Int J Mol Sci 2017;18. doi:10.3390/ijms18091868. [Epub ahead of print: 29 Aug 2017].

8 Schuster SJ, Svoboda J, Chong EA, et al. Chimeric antigen receptor T cells in refractory B-cell lymphomas. $N$ Engl J Med 2017;377:2545-54.

9 Park JH, Rivière I, Gonen M, et al. Long-Term follow-up of CD19 CAR therapy in acute lymphoblastic leukemia. N Engl J Med 2018;378:449-59.

10 Neelapu SS, Locke FL, Bartlett NL, et al. Axicabtagene Ciloleucel CAR T-cell therapy in refractory large B-cell lymphoma. N Engl J Med 2017;377:2531-44.

11 O'Connor BP, Raman VS, Erickson LD, et al. Bcma is essential for the survival of long-lived bone marrow plasma cells. J Exp Med 2004;199:91-8.

12 Carpenter RO, Evbuomwan MO, Pittaluga S, et al. B-Cell maturation antigen is a promising target for adoptive T-cell therapy of multiple myeloma. Clin Cancer Res 2013;19:2048-60.

13 Novak AJ, Darce JR, Arendt BK, et al. Expression of BCMA, TACI, and BAFF-R in multiple myeloma: a mechanism for growth and survival. Blood 2004:103:689-94.

14 Perez-Amill L, Suñe G, Antoñana-Vildosola A, et al. Preclinical development of a humanized chimeric antigen receptor against $\mathrm{B}$ cell maturation antigen for multiple myeloma. Haematologica 2020;106:173-84.

15 Zhao W-H, Liu J, Wang B-Y, et al. A phase 1, open-label study of LCAR-B38M, a chimeric antigen receptor $T$ cell therapy directed against $B$ cell maturation antigen, in patients with relapsed or refractory multiple myeloma. J Hematol Oncol 2018;11:141.

16 Raje N, Berdeja J, Lin Y, et al. Anti-BCMA CAR T-cell therapy bb2121 in relapsed or refractory multiple myeloma. $N$ Engl $\mathrm{J}$ Med 2019;380:1726-37.

17 Shah N, Li L, McCarty J, et al. Phase I study of cord bloodderived natural killer cells combined with autologous stem cell transplantation in multiple myeloma. $\mathrm{Br} J$ Haematol 2017:177:457-66.

18 Shah N, Martin-Antonio B, Yang H, et al. Antigen presenting cellmediated expansion of human umbilical cord blood yields log-scale expansion of natural killer cells with anti-myeloma activity. PLoS One 2013;8:e76781.

19 Tang X, Yang L, Li Z, et al. First-In-Man clinical trial of car NK92 cells: safety test of CD33-CAR NK-92 cells in patients with relapsed and refractory acute myeloid leukemia. Am J Cancer Res 2018;8:1083-9.

20 Martín-Antonio B, Suñe G, Najjar A, et al. Extracellular NK histones promote immune cell anti-tumor activity by inducing cell clusters through binding to CD138 receptor. J Immunother Cancer 2019;7:259

21 Castella M, Boronat A, Martín-lbáñez R, et al. Development of a novel anti-CD19 chimeric antigen receptor: a paradigm for an affordable CAR T cell production at academic institutions. Mol Ther Methods Clin Dev 2019;12:134-44.

22 Bari R, Granzin M, Tsang KS, et al. A Distinct Subset of Highly Proliferative and Lentiviral Vector (LV)-Transducible NK Cells Define a Readily Engineered Subset for Adoptive Cellular Therapy. Front Immunol [Internet]. Frontiers, 201910. Available from. Available: https://www.frontiersin.org/articles/10.3389/fimmu.2019.02001/full [Accessed 03 Mar 2021].

23 Mingari MC, Gerosa F, Carra G, et al. Human interleukin-2 promotes proliferation of activated $B$ cells via surface receptors similar to those of activated T cells. Nature 1984;312:641-3.

24 Schultz M, Clarke SH, Arnold LW, et al. Disrupted B-lymphocyte development and survival in interleukin-2-deficient mice. Immunology 2001;104:127-34.

25 Chernyshov VP, Dons'koi BV, Sudoma IO, et al. Comparison of T and NK lymphocyte subsets between human endometrial tissue and peripheral blood. Cent Eur J Immunol 2019;44:316-21.

26 Martin MD, Badovinac VP. Defining Memory CD8 T Cell. Front Immunol [Internet]. Frontiers, 2018. Available: https://www. frontiersin.org/articles/10.3389/fimmu.2018.02692/full [Accessed 23 Mar 2021].
27 Battram AM, Bachiller M, Martín-Antonio B. Senescence in the development and response to cancer with immunotherapy: a doubleedged sword. Int J Mol Sci 2020;21:4346.

28 Dirks J, Tas H, Schmidt T, et al. PD-1 analysis on CD28(-) CD27(-) CD4 T cells allows stimulation-independent assessment of CMV viremic episodes in transplant recipients. Am J Transplant 2013;13:3132-41.

29 Tanaskovic S, Price P, French MA, et al. Impaired upregulation of the costimulatory molecules, CD27 and CD28, on $\mathrm{CD}^{+}{ }^{+} \mathrm{T}$ cells from HIV patients receiving ART Is associated with poor proliferative responses. AIDS Res Hum Retroviruses 2017;33:101-9.

30 Neurohr GE, Terry RL, Lengefeld J, et al. Excessive cell growth causes cytoplasm dilution and contributes to senescence. Cell 2019;176:e18:1083-97.

31 Hay KA, Turtle CJ. Chimeric antigen receptor (CAR) T cells: lessons learned from targeting of CD19 in B-cell malignancies. Drugs 2017;77:237-45.

32 Rafiq S, Hackett CS, Brentjens RJ. Engineering strategies to overcome the current roadblocks in car T cell therapy. Nat Rev Clin Oncol 2020;17:147-67.

33 Liu E, Ang SOT, Kerbauy L, et al. GMP-Compliant universal antigen presenting cells (UAPC) promote the metabolic fitness and antitumor activity of armored cord blood CAR-NK cells. Front Immunol 2021:12:626098.

34 Curti A, Ruggeri L, D'Addio A, D'Addio A, et al. Successful transfer of alloreactive haploidentical KIR ligand-mismatched natural killer cells after infusion in elderly high risk acute myeloid leukemia patients. Blood 2011;118:3273-9.

35 Bachanova V, Cooley S, Defor TE, et al. Clearance of acute myeloid leukemia by haploidentical natural killer cells is improved using IL-2 diphtheria toxin fusion protein. Blood 2014;123:3855-63.

36 Curti A, Ruggeri L, Parisi S, et al. Larger size of donor alloreactive NK cell repertoire correlates with better response to NK cell immunotherapy in elderly acute myeloid leukemia patients. Clin Cancer Res 2016;22:1914-21.

37 Karadimitris A. Cord blood CAR-NK cells: favorable initial efficacy and toxicity but durability of clinical responses not yet clear. Cancer Cell 2020;37:426-7.

38 Ali SA, Shi V, Maric I, et al. T cells expressing an anti-B-cell maturation antigen chimeric antigen receptor cause remissions of multiple myeloma. Blood 2016;128:1688-700.

39 Khatua S, Cooper LJN, Sandberg DI, et al. Phase I study of intraventricular infusions of autologous ex vivo expanded NK cells in children with recurrent medulloblastoma and ependymoma. Neuro Oncol 2020;22:1214-25.

40 Waggoner SN, Cornberg M, Selin LK, et al. Natural killer cells act as rheostats modulating antiviral T cells. Nature 2011;481:394-8.

41 Peppa D, Gill US, Reynolds G, et al. Up-regulation of a death receptor renders antiviral T cells susceptible to NK cell-mediated deletion. J Exp Med 2013;210:99-114.

42 Schuster IS, Wikstrom ME, Brizard G, et al. TRAIL+ NK cells control CD4+ T cell responses during chronic viral infection to limit autoimmunity. Immunity 2014;41:646-56.

43 Habib S, El Andaloussi A, Hisham A, et al. NK cell-mediated regulation of protective memory responses against intracellular ehrlichial pathogens. PLoS One 2016;11:e0153223.

44 Marcenaro E, Ferranti B, Falco M, et al. Human NK cells directly recognize mycobacterium bovis via TLR2 and acquire the ability to kill monocyte-derived DC. Int Immunol 2008;20:1155-67.

45 Littwitz-Salomon E, Malyshkina A, Schimmer S, et al. The Cytotoxic Activity of Natural Killer Cells Is Suppressed by IL-10+ Regulatory T Cells During Acute Retroviral Infection. Front Immunol 2018;9:9

46 Bachanova V, Sarhan D, DeFor TE, et al. Haploidentical natural killer cells induce remissions in non-Hodgkin lymphoma patients with low levels of immune-suppressor cells. Cancer Immunol Immunother 2018;67:483-94.

47 Björklund AT, Carlsten M, Sohlberg E, et al. Complete remission with reduction of high-risk clones following haploidentical NK-cell therapy against MDS and AML. Clin Cancer Res 2018;24:1834-44.

48 Vivier E, Tomasello E, Baratin M, et al. Functions of natural killer cells Nat Immunol 2008;9:503-10.

49 Russo A, Oliveira G, Berglund S, et al. Nk cell recovery after haploidentical HSCT with posttransplant cyclophosphamide: dynamics and clinical implications. Blood 2018;131:247-62.

50 Bluhm J, Kieback E, Marino SF, et al. Car T cells with enhanced sensitivity to $B$ cell maturation antigen for the targeting of $B$ cell non-Hodgkin's lymphoma and multiple myeloma. Mol Ther 2018;26:1906-20.

51 Salter Al, Ivey RG, Kennedy JJ, et al. Phosphoproteomic analysis of chimeric antigen receptor signaling reveals kinetic and quantitative differences that affect cell function. Sci Signal 2018;11. doi:10.1126/ scisignal.aat6753. [Epub ahead of print: 2108 2018]. 
52 Lucas F, Pennell M, Huang Y, et al. T cell transcriptional profiling and immunophenotyping uncover LAG3 as a potential significant target of immune modulation in multiple myeloma. Biol Blood Marrow Transplant 2020;26:7-15.

53 Lozano E, Mena M-P, Díaz T, et al. Nectin-2 expression on malignant plasma cells is associated with better response to TIGIT blockade in multiple myeloma. Clin Cancer Res 2020;26:4688-98.
54 Castella M, Fernández de Larrea C, Martín-Antonio B. Immunotherapy: a novel era of promising treatments for multiple myeloma. Int J Mol Sci 2018;19. doi:10.3390/ijms19113613. [Epub ahead of print: 15 Nov 2018].

55 Zelle-Rieser C, Thangavadivel S, Biedermann R, et al. T cells in multiple myeloma display features of exhaustion and senescence at the tumor site. J Hematol Oncol 2016;9:116. 